\title{
Dynamics and flow characterization of liquid fountains produced by light scattering
}

\author{
Hugo Chesneau, ${ }^{*}$ Julien Petit, ${ }^{*}$ Hamza Chraïbi ${ }^{\dagger}$ and Jean-Pierre Delville ${ }^{\ddagger}$ \\ Univ. Bordeaux, CNRS, LOMA, UMR 5798, F-33405 Talence, FRANCE
}

(Dated: December 19, 2019)

\begin{abstract}
We present a detailed study of the dynamics of soft liquid interfaces undergoing viscous stresses due to bulk flows generated by momentum transfer from light to a turbid liquid. Using a continuous laser wave, light-induced flow is observed and analyzed through the deformability of very soft interfaces up to instability and jet formation. These dynamics are investigated experimentally and numerically below and above the interface instability threshold. Below instability, we show that the dynamics of the interface deformation at short time scale does not vary with the parameters of the laser excitation. We confirm that the mechanism responsible for the interface deformation is a non-local effect associated to the viscous stress induced by the bulk flow. Then, we characterize the jetting instability regarding the field velocity within the jet, the jet radius and the fluid flow rate. A satisfying agreement is obtained when comparing quantitatively experimental results and numerical predictions. Our investigation illustrates how light can induce a bulk flow in a turbid liquid, such as a suspension, and how this flow can be used to deform an interface and produce well-controlled liquid jets.
\end{abstract}

Light is able to set fluids in movement transiently or permanently by transfer of energy or momentum. The most famous example is thermal Marangoni flow (called optocapillarity when induced by light) due to heat transfer to the interface when a liquid layer absorbs light at the used optical wavelength. It was widely studied experimentally, theoretically and numerically as it is easily induced by a local laser-heating [1-4]. With the development of microfluidic toolboxes on the one hand, and the enhancement of the surface to volume coupling contributions with miniaturization, on the other hand, many efforts were concentrated towards the developments of optically induced surface-tensiondriven flows at the microscale. Among these examples, one can cite the control of the spreading of films [5], the production of droplets [6], the manipulation of jets [7], or the actuation of particles floating at a free surface [8]. The investigation of flows induced by laser heating was further extended in bulk using absorbing suspensions [9-11] or at interfaces considering light-sensitive surfactants [12].

However, as shown in the present study, light may also transfer momentum isothermally to produce flows. We concentrate here on this totally different mechanism where thermal effects are irrelevant. For this purpose the fluid system at work, a phase-separated liquid mixtures close to criticality, has an extremely weak optical absorption at the used optical wavelength making thermal effects negligible. Pure transfer of light momentum to a fluid can be set in a non-absorbing liquid suspension and the mechanism is the following : when the liquid is constituted by randomly distributed sub-wavelength particles in suspension or density fluctuations as in critical fluids, these refractive index heterogeneities scatter the incident light beam which eventually loses forward momentum during its propagation in the medium ; this phenomenon corresponds to the well-known critical opalescence in critical phenomena. As a consequence, momentum conservation produces a density force, called scattering force, that sets the liquid in permanent motion. As optical absorption is discarded, this density force should be only proportional to the wave momentum $(n I / c) z$ and to the beam attenuation $\varpi=(-1 / I) d I / d z$ due to the liquid turbidity, with $n$ the refractive index of the medium, $I$ the intensity of the laser beam and $c$ the light celerity ; $\varpi$ may also be called extinction coefficient when dealing with sub-wavelength particle suspensions. The expected scattering force density $f^{\text {scatt }}(r)$ is then proportional to $(\varpi n I / c) \boldsymbol{z}$ for $\varpi L \ll 1$ where $\mathrm{L}$ is the thickness of the sample. As illustrated in a recent review [13], this scattering force at the level of the suspended particles is at the origin of many theoretical and experimental works dedicated to particle manipulations in fluids. A similar physical approach was also described to control the advection of small particles at free surfaces efficiently [14]. In the opposite case Savchenko et al. [10], theoretically showed for the first time that it should be possible to induce a convective flow, by transfer of momentum from light to a liquid crystal. Skipetrov et al. $[15,16]$ demonstrated that multiple scattering events also drive bulk flow and measured the scatterer velocity by determining the temporal autocorrelation function of the scattered electromagnetic field. Later Casner and Delville [17] strongly deformed very soft fluid/fluid interfaces in near critical thermodynamic conditions using the optical radiation pressure. They evidenced a jetting instability at the tip of the interface deformation with droplet production, both unexplained by the surface radiation pressure which cannot actuate steady flows. In the absence of optical absorption, this effect was attributed to light scattering [18]

\footnotetext{
* These authors contributed equally to this work.

$\dagger$ hamza.chraibi@u-bordeaux.fr

¥ jean-pierre.delville@u-bordeaux.fr
} 
as the turbidity of the involved fluid system diverges close to a critical point. This was confirmed analytically in the weak interface deformation regime by considering the viscous stress exerted on the interface by the resulting bulk flow. This scattering effect was further explored theoretically and experimentally in stationary conditions to characterize the induced bulk flow [19]. Nonetheless, in this last study, bulk scattering force and interfacial radiation pressure effects were collaborating in the same direction, making difficult to discriminate the importance of each mechanism on the resulting interface instability. Consequently a numerical investigation on pure scattering density effects, based on a Boundary Element Method (BEM), in the small deformation regime (i.e. when the amplitude of the deformed interface is smaller than the beam waist) was developed in order to grasp the properties of the induced bulk flow in permanent conditions [20]. Indeed, to discriminate between radiation pressure and scattering bulk flow effects, Chraïbi et al. [21] investigated the resulting jetting instability when the radiation pressure and the scattering force act in opposite directions. This approach gave the opportunity to observe for the first time, how the interface stress induced by the bulk flow due to the scattering force alone can deform an interface, up to instability and jetting. Nonetheless, these numerical investigations were limited to the features of the interface without characterizing the flows.

The goal of the present work is to investigate the dynamic features of the flow in terms of interface velocity and fluid flow rate as a function of the driving parameters which are the beam power and fluid properties. We describe first mechanical forces induced by the laser beam and the experimental setup. Then we study the dynamics of the interface in the small deformation regime when scattering forces remain too weak to destabilize this interface. In a third part, we analyze the dynamics at and beyond interface instability and we characterize the properties of the induced jets in terms of velocity field, radius and flow rate as a function of the excitation. A summary of the physical properties and of the dimensionless numbers used in the present study is presented in the appendix B. This investigation gives a first opportunity to quantitatively compare experiments and numerical simulations in order to understand how light can trigger flow and jetting in non-absorbing liquid suspensions ; the detailed calculation of the scattering force resulting from the momentum transfer of the incident light to a turbid media in the Rayleigh scattering regime (i.e. for particles or refractive index fluctuations very small compared to the optical wavelength) is presented in the appendix A.

We may note at the end of this introduction that, even if our investigation is entirely dedicated to liquid flows induced by mechanical effects of non-absorbing light, acoustic radiation pressure and streaming, historically known well before the laser invention, can as well deform interfaces and produce flows. While mechanisms may be slightly different, strictly speaking richer in acoustics because the optical impedance is usually reduced to refractive index contrast, the spirit is exactly the same. Interface deformation by the radiation pressure was nicely demonstrated in acoustics by Hertz and Mende [22] and much later with lasers by Ashkin and Dziedzic [23]. Thus acoustically-driven flow $[24,25]$, interface instability and jetting [26], and droplet production [27, 28] have for a long time been a subject of interest, unveiling universal behaviors triggered by radiation pressure regardless of the nature of the exciting wave, whether mechanical and longitudinal (acoustics) or electromagnetic and transverse (optics).

\section{LIGHT-INDUCED FORCES AND EXPERIMENTAL SETUP}

\section{A. Mechanical effects of light in bulk and on interface}

\section{Bulk flows driven by light scattering in turbid liquids}

As qualitatively mentioned in the introduction, the density force $\boldsymbol{f}^{\text {scatt }}(r)$ should be proportional to the wave momentum, $(n I / c) \boldsymbol{z}$, and to the beam attenuation $\varpi=(-1 / I) d I / d z$, here corresponding to the turbidity since optical absorption is discarded. The main steps of its calculation are the following : first, we need to determine the light field scattered by the liquid heterogeneities. It linearly depends on the structure factor $S=\left\langle|\delta \rho|^{2}\right\rangle$ that describes the correlation between density fluctuations $\delta \rho$. Moreover $S$ is calculated within the Ornstein-Zernike approximation, usually considered for light scattering in critical fluids. Then, as illustrated in the appendix A, we can deduce the fraction of scattered intensity $I\left(q, R_{o}\right) / I_{0}$ at a distance $R_{o}$ from the small scattering volume $V$ of interest :

$$
\frac{I\left(q, R_{o}\right)}{I_{0}}=\left(\frac{\pi^{2}}{\lambda_{0}^{4}}\right)\left(\frac{\partial \epsilon}{\partial \Phi}\right)^{2} k_{B} T \chi_{T}\left[\frac{1}{1+(q \xi)^{2}}\right]\left(\frac{V}{R_{o}^{2}}\right) \sin ^{2} \phi .
$$

In equation (1), $I_{0}$ is the incident beam intensity, $q=\left|\boldsymbol{k}_{i}-\boldsymbol{k}_{s}\right|=2 k_{i} \sin \theta / 2$ where $\boldsymbol{k}_{i}$ and $\boldsymbol{k}_{s}$ are respectively the incident and the scattered optical wave-vectors, $\lambda_{0}$ is the optical wavelength in vacuum and $\theta$ is the scattering angle. $\xi$ and $\Phi$ are the size and the concentration of the scatterers, and $\chi_{T}$ is the isotherm compressibility. $(q \xi)^{2}$ can as well 
be written as $(q \xi)^{2}=\alpha(1-\cos \theta)$ with $\alpha=2\left(2 \pi \sqrt{\epsilon_{r}} \xi / \lambda_{0}\right)^{2}$ and $\epsilon_{r}=n^{2}$. Finally, $\phi$ represents the angle between the polarization of the incident wave and the scattering direction $\boldsymbol{k}_{s}$. When an incident photon, of momentum $\hbar \boldsymbol{k}_{i}$ is elastically scattered by a refractive index heterogeneity with an angle $\theta$, its momentum in the direction $\boldsymbol{k}_{i}$ decreases by a factor $\hbar k_{i}(1-\cos \theta)$ and the difference is transferred to the fluid, giving birth to the scattering density force $\boldsymbol{f}^{\text {scatt }}(r)$ that sets the fluid in movement. Integration over a sphere of radius $R_{o}$ of $\hbar k_{i}(1-\cos \theta)$ corrected by the fraction of scattered intensity (equation (1)) finally gives the average light momentum lost in the direction of propagation $\boldsymbol{k}_{i}$. Then, from Newton's second law, we deduce the corresponding scattering force density :

$$
\boldsymbol{f}^{\text {scatt }}(r)=\frac{n I(r)}{c}\left(\frac{\pi^{3}}{\lambda_{0}^{4}}\right)\left(\frac{\partial \epsilon_{r}}{\partial \Phi}\right)^{2} k_{B} T \chi_{T} f(\alpha) \frac{\boldsymbol{k}_{i}}{\left|\boldsymbol{k}_{i}\right|}
$$

where $f(\alpha)=1 / \alpha^{4}\left[8 / 3 \alpha^{3}+2 \alpha^{2}+2 \alpha-\left(2 \alpha^{2}+2 \alpha+1\right) \ln (1+2 \alpha)\right]$. This density force is, as expected, proportional to the wave momentum and to the turbidity $\varpi=\left(\frac{\pi^{3}}{\lambda_{0}^{4}}\right)\left(\frac{\partial \epsilon_{r}}{\partial \Phi}\right)^{2} k_{B} T \chi_{T} f(\alpha)$. At low Reynolds number, the Stokes equation easily sets an order of magnitude of the resulting axial velocity : $v^{\text {scatt }}=\pi \omega_{0}^{2} f^{\text {scatt }} / \eta$, where $\eta$ is the shear viscosity and $\omega_{0}$ is the laser beam waist. Typical values of $v^{\text {scatt }}$ in our near-critical systems are in the range $v^{\text {scatt }}=\left(10^{-5}-10^{-4}\right) \mathrm{m} \mathrm{s}^{-1}$. When directed normal to a fluid interface, this scattering velocity induces in turn a normal viscous stress $\eta \partial v / \partial z \sim \eta v / L_{c} \sim \sigma / L_{c}$ where $L_{c} \in[0.1-1] \mathrm{mm}$ is the typical lengthscale associated with the flow which scales like the smallest dimension of the container [20]. In our two-fluid systems, we typically get $\eta \partial v / \partial z \sim\left(10^{-5}-10^{-3}\right) \mathrm{Pa}$. As a consequence, observation of micrometric interface deformations by scattering forces requires a Laplace pressure (and buoyancy) of the same order of magnitude. Such a balancing condition suggests use of low interfacial tension systems and explains the choice of phase-separated near-critical mixture considered in the present investigation since tension $\sigma$ as low as $\left(10^{-8}-10^{-7}\right) \mathrm{N} \mathrm{m}^{-1}$ can be achieved.

\section{Optical radiation pressure at fluid interfaces}

As soon as a fluid interface is present and interface deformation is under investigation, a second unavoidable mechanical effect of light is expected to occur experimentally : interface deformation by the optical radiation pressure. If this interface separates two dielectric liquids of different index of refraction $n_{i, t}$ (subscripts $i$ and $t$ refer here to incidence and transmission), which is almost always the case, then the photon momentum $p=\hbar k_{i}=n_{i} h \nu / c$, where $h \nu$ is the photon energy, is not conserved when traveling from one liquid to the other. The resulting mismatch gives birth to a radiation pressure applied to the interface in order to conserve momentum. This optical radiation pressure is a function of the incident and transmitted angles $\theta_{i}$ and $\theta_{t}$ at the interface. The elementary variation of the normal and tangent momentum components at the interface [29] is obtained by counting (i) the momentum given to the interface by an incident photon (ii) the momentum picked to the interface by a reflected photon, and finally (iii) the momentum picked to the interface by a transmitted photon. Then, it can be deduced that (i) in the absence of dissipation, there is no momentum transfer parallel to the interface and (ii) for a laser wave incident with an angle $\theta_{i}$, the expression of the optical radiation pressure is :

$$
\boldsymbol{\Pi}_{R a d}=n_{i} \cos ^{2} \theta_{i}\left[1+R\left(\theta_{i}, \theta_{t}\right)-\frac{\tan \theta_{i}}{\tan \theta_{t}} T\left(\theta_{i}, \theta_{t}\right)\right] \frac{I}{c} \boldsymbol{n}
$$

Where $\boldsymbol{n}$ is a unit vector normal to the interface and, $R\left(\theta_{i}, \theta_{t}\right)$ and $T\left(\theta_{i}, \theta_{t}\right)=1-R\left(\theta_{i}, \theta_{t}\right)$ are the classical reflection and transmission Fresnel coefficients in electromagnetic energy. By developing the expression of $R\left(\theta_{i}, \theta_{t}\right)$, it appears that $\boldsymbol{\Pi}_{R a d}$ is always normal to the interface and directed towards the liquid of smallest index of refraction whatever the direction of beam propagation ; in our case from fluid 2 to fluid 1 (see figure 1). The stationary height $h(r)$ of the interface deformed by the optical radiation pressure can as well be found by balancing buoyancy $\left(\rho_{1}-\rho_{2}\right) g h(r)$ and Laplace pressure $\Pi_{\text {Laplace }}(r)=-\sigma \kappa(r)$ on the one hand, and radiation pressure $\Pi_{R a d}(r)$ on the other hand. At normal incidence, on the beam axis and for close indices of refraction $\Pi_{r a d} \approx\left(n_{2}-n_{1}\right) I_{0} / c$, where $I_{0}=2 P /\left(\pi \omega_{0}^{2}\right)$ is the beam intensity at $r=0$ and $P$ and $\omega_{0}$ are respectively the injected power and beam waist. For the typical values of refractive index $\left(n_{2}-n_{1}\right)=10^{-2}-10^{-3}$, power $P=1 \mathrm{~W}$ and beam waist $\omega_{0}=10^{-5} \mathrm{~m}$, we find $\Pi_{\text {rad }} \approx\left(10^{-2}-10^{-1}\right) \mathrm{Pa}$. While the characteristic length of the flow induced by the scattering force is $L_{c}$, i.e. the smallest size of the container, the one characterizing the radiation pressure is $\omega_{0}$ the laser beam waist as $I\left(r>\omega_{0}\right) \approx 0$ and thus $\Pi_{\text {rad }}\left(r>\omega_{0}\right) \approx 0$.

\section{Induced effects of the involved forces on the interface and required experimental system.}

Four main conclusions can then be advanced from the previous orders of magnitude. The first two are related to the mechanical effects of light. (i) Radiation pressure cannot be avoided experimentally when investigating scattering 

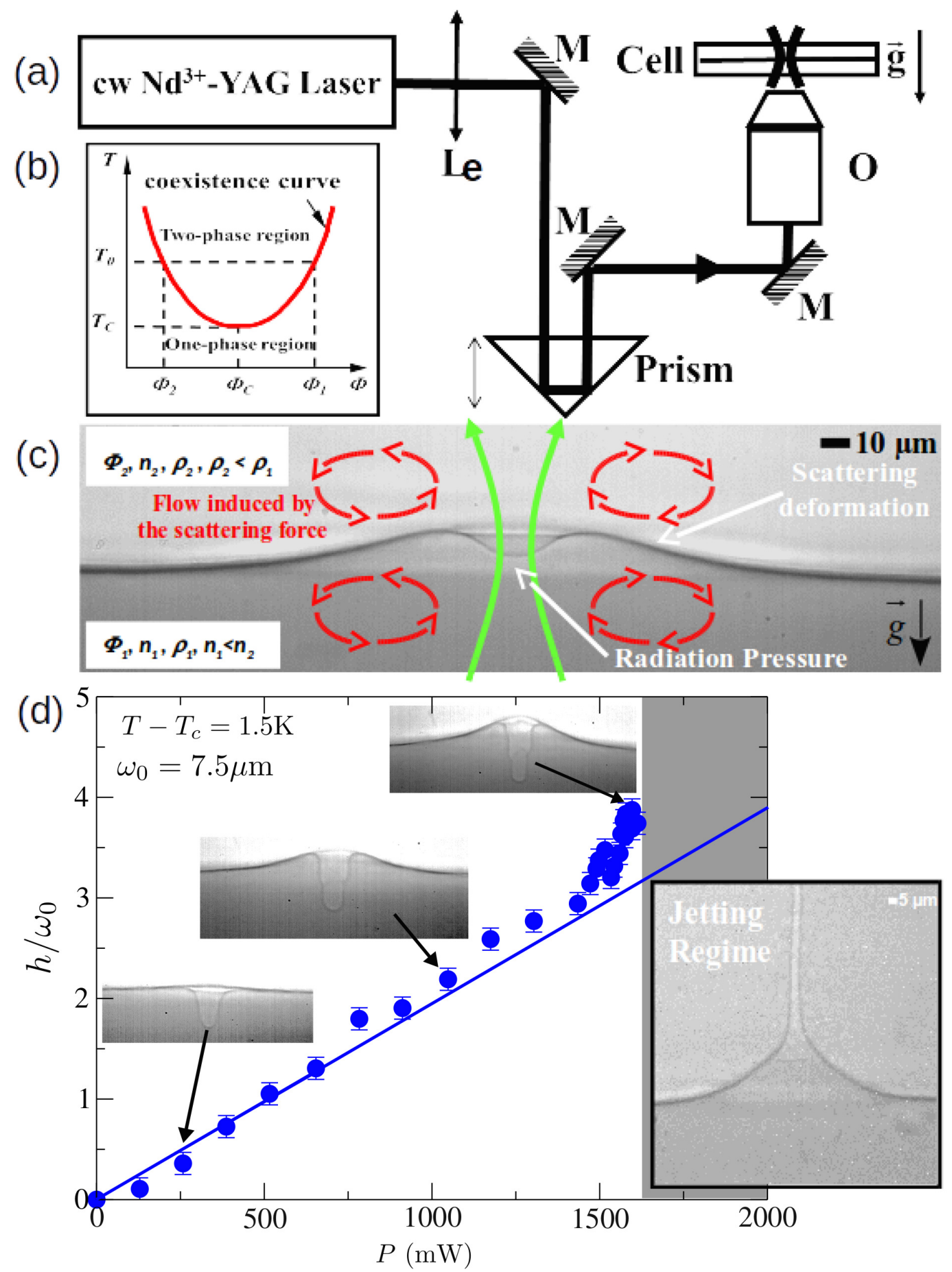

FIG. 1. (a) Illustration of the experimental setup. $\mathrm{L}_{\mathrm{e}}$ is a convergent lens with a focal length $F e=80 \mathrm{~cm}, \mathrm{M}$ are mirrors and $\mathrm{O}$ is an Olympus 10X objective with long working distance. (b) Schematic coexistance curve of the phase-separated near-critical microemulsion. (c) Picture of a deformed interface by a laser beam. It shows the deformation inherent to radiation pressure (downward in the center of the picture), the one induced by the scattering force, and the emergence of a shoulder due to antagonist effects. The red arrows represent the eddies induced by the scattering force (d) Height variation of the shoulder with the injected power. The gray region represents the jetting regime. 
effects on interfaces because classically the two phases in contact have different index of refraction. (ii) As radiation pressure is expected to be larger than the normal viscous stresses due to scattering forces, even at low refractive index contrast, the best way to investigate scattering effects thus needs an experimental configuration where both effects act in opposite direction to partly disentangle them. The two other conclusions are important for setting experimental conditions. (i) Turbid liquids are necessary for the observation of scattering effects, either real suspensions or fluctuating systems. (ii) Considering the order of magnitude of the expected induced normal viscous stress on the interface, a balance with the Laplace pressure (and possibly buoyancy) necessarily requires very low interfacial tension systems. It is not only sufficient to use a turbid liquid such as a suspension but it also has to be easily deformable. Fulfillment of both conditions led us to choose phase-separated liquid mixtures close to criticality described in the following part.

\section{B. Phase-separated near-critical fluid system and experimental setup}

To investigate light scattering stresses on liquid interfaces, we used the near-critical two-phase equilibrium state of a micellar phase of a microemulsion. The microemulsion is composed of water, oil (toluene), surfactant (sodium dodecyl sulfate, SDS), and co-surfactant (n-butanol-1). At low ratio of water and surfactant, thermodynamic equilibrium leads to the formation of a supramolecular binary liquid mixture composed of a suspension of water nanodroplets coated by a shell of surfactant, the micelles, dispersed in an oil continuum ; soluble on both water and oil, the co-surfactant is used for reducing the hydrophilic/lipophilic balance of SDS and thus allowing the formation of micelles. For the chosen composition (mass fractions in \%wt: water, 9\%, toluene, $79 \%$, SDS, $4 \%$, butanol, $17 \%$ ), the micelles radius is about $4 \mathrm{~nm}[30]$; this value is small enough to keep the mixture slightly translucent at room temperature. As for any liquid mixture, our micellar solution presents a line of liquid-liquid critical points, here associated with a reverted coexistence curve [31] ; see figure 1b. It has also been demonstrated that this microemulsion belongs to the universality class $(\mathrm{d}=3, \mathrm{n}=1)$ of the Ising model [32], implying that mechanical effects of light analyzed in such a system are expected to happen for any isotropic liquid in the same way. At the chosen composition, the critical temperature is $T_{c} \simeq 35^{\circ} \mathrm{C}$. Above $T_{c}$, this mixture separates in two micellar phases of concentrations $\Phi_{1}$ and $\Phi_{2}$ (respectively rich and poor in micelles), illustrated by the schematic phase diagram shown in figure 1b. Fluid 1 and 2 denote respectively the bottom and top phases as illustrated in figure 1c. As for any system close to criticality, some properties, in the two-phase region, present divergent scaling laws or vanishing behaviors that are characteristic of second-order phase transition. Here the order parameter is the contrast in concentration $\left(\Phi_{1}-\Phi_{2}\right)$. Important quantities for the present investigation are :

(i) The correlation length of density fluctuations in the two-phase region which is involved in the fraction of scattered intensity and then in the scattering density force,

$$
\xi^{-}=\xi_{0}^{-}\left(\frac{T-T_{c}}{T_{c}}\right)^{-0.63}, \text { with } \xi_{0}^{-}=2 \mathrm{~nm} .
$$

(ii) The susceptibility involved in the turbidity, and then in the scattering density force,

$$
\chi^{-}=\chi_{0}^{-}\left(\frac{T-T_{c}}{T_{c}}\right)^{-1.24} \text {, with } \chi_{0}^{-}=1.344 \times 10^{-6} \mathrm{~Pa}^{-1} .
$$

(iii) The interfacial tension involved in the Laplace pressure,

$$
\sigma=\sigma_{0}\left(\frac{T-T_{c}}{T_{c}}\right)^{1.26}, \text { with } \sigma_{0}=5 \times 10^{-5} \mathrm{~N} \mathrm{~m}^{-1} .
$$

(iv) The density contrast involved in buoyancy,

$$
\left(\rho_{1}-\rho_{2}\right)=\Delta \rho_{0}\left(\frac{T-T_{c}}{T_{c}}\right)^{0.325}, \text { with } \Delta \rho_{0}=53.625 \mathrm{~kg} \mathrm{~m}^{-3} .
$$

(v) the contrast of index of refraction involved in radiation pressure,

$$
\left(n_{1}-n_{2}\right)=\Delta n_{0}\left(\frac{T-T_{c}}{T_{c}}\right)^{0.325}, \text { with } \Delta n_{0}=-0.0451
$$

And finally (vi) the capillary length involved in the optical Bond number $B o=\left(\omega_{0} / l_{c}\right)^{2}$,

$$
l_{c}=\sqrt{\frac{\sigma}{\left(\rho_{1}-\rho_{2}\right) g}}=l_{c 0}\left(\frac{T-T_{c}}{T_{c}}\right)^{0.47}, \text { with } l_{c 0}=3.1 \times 10^{-4} \mathrm{~m} .
$$


Also of importance is the shear viscosity of the two phases $\eta_{i}(T)=[1.46-0.014(T-237)]\left(1+2.5 \Phi_{i}\right) 10^{-3} \mathrm{Pas}$ which depends on the concentration of the micellar phase $\Phi_{i=1,2}=\Phi_{0} \pm \Delta \Phi_{0} / 2\left(\left(T-T_{c}\right) / T_{c}\right)^{0.325}$ with $\Phi_{0}=0.11$ and $\Delta \Phi_{0}=0.275$. These scaling also show another important advantage in using such fluid systems : the abovementioned properties can be tuned continuously just by changing the temperature of the system. In the present study, we chose $\Delta T=T-T_{c}=0.5,1.5,1.9 \mathrm{~K}$. Finally, the optical absorption of the mixture $\alpha_{a} \approx 3 \times 10^{-4} \mathrm{~cm}^{-1}$ at the used wavelength $\left(\lambda_{0}=532 \mathrm{~nm}\right.$ in vacuum) is weak enough, to discard for the investigated temperatures, the laser heating side effects such as thermo-convection and Marangoni flows.

The experimental setup is illustrated in figure 1a. It consists in focusing a vertical laser beam on the horizontal fluid interface of the two-phase sample contained in a thermally controlled fused quartz cell $\left(40 \times 10 \times 2 \mathrm{~mm}^{3}\right)$. The light beam is provided by a continuous wave frequency doubled $\mathrm{Nd}^{3+}-\mathrm{YAG}$ laser in the $\mathrm{TEM}_{00}$ mode. As the beam intensity, involved in both the scattering force and the radiation pressure, depends on both the beam power and waist, the setup allows for the variation of both quantities. Power is directly controlled by the power supply of the laser and the optical transmission of the setup. For the waist variation, we use the following scheme. The lens $\mathrm{L}_{\mathrm{e}}$ forms a first waist along the optical path and moving the prism Pr allows variation of the optical path between $L_{e}$ and the microscope objective O (Olympus 10X, long working distance) used to focus the beam at the interface. Using a focal lens $F_{e}=80 \mathrm{~cm}$, the range of accessible beam waists in the sample is $\omega_{0} \sim(3-14) \mu \mathrm{m}$. With these values, the corresponding Rayleigh length, i.e. the length over which the beam can be assumed to be almost cylindrical, is always larger than $100 \mu \mathrm{m}$, thus allowing numerical simulations to be confidently performed using cylindrical non-diverging laser waves. Note finally that the beam waist altitude inside the sample varies with the optical path between the lens $\mathrm{L}_{\mathrm{e}}$ and the objective $\mathrm{O}$, so the cell is mounted on a vertical translation stage to precisely set the chosen beam waist on the interface ; this is done by observing the beam propagation in the sample using its scattering by the micelles (or the density fluctuations). As the index of refraction of the phase $\Phi_{1}$ is smaller than that of $\Phi_{2}$ on the one hand, and since we want to set opposing scattering and radiation pressure effects on the other hand, the laser beam is chosen to be incident from the phase $\Phi_{1}$ and propagates upward. The temperature $T$ is controlled with a stability of $\pm 0.05 \mathrm{~K}$ using a PID. An example of deformation of the interface separating the two phases $\Phi_{1}$ and $\Phi_{2}$ above $T_{c}$ is illustrated in figure 1c ; both scattering and radiation pressure effects are observed, acting in opposite directions as expected. This interface is illuminated by a white light source and deformations are observed using a large-chip high-speed CMOS camera. To prevent saturation of the chip, the field scattered in the sample is partially or completely suppressed using colored glass filters in front of the camera. A typical example of experiment is presented in figure 1d. By increasing the beam power, for given waist and $T-T_{c}$, the interface is increasingly deformed in both vertical directions : (i) due to the refractive index contrast between the two phases, the radiation pressure produces a deformation downward with a typical radial size comparable to the beam diameter, and (ii) a much wider deformation in the direction of propagation, i.e. upward, due to scattering forces on both liquid phases ; this larger wideness is due to scattering effects and not only depends on beam intensity but also on hydrodynamic boundary conditions. Note also on snapshots that interface shapes due to radiation pressure become nonlinear when increasing the beam power [33]. As scattering and radiation pressure effects are chosen to be antagonist in order to disentangle them as much as possible, the curve presented in figure 1d corresponds to the shoulder height where the contribution of scattering flows versus radiation pressure stresses is the largest. For the chosen conditions, it appears that this shoulder height starts to behave nonlinearly for incident power between $P=0.5 \mathrm{~W}$ and $P=1.0 \mathrm{~W}$. Above this range, the height increases more rapidly and eventually the interface deformation becomes unstable at the shoulder location for some well-defined threshold power, giving birth to an off-axis stable jet.

In the following part, we describe the physical system and the numerical method used.

\section{PHYSICAL MODEL}

To solve numerically the deformation of an initially flat interface by the scattering force, we use a boundary element method [20]. The Reynolds number being small $\left(R e \approx 10^{-4}[19]\right)$ we solve the Stokes equation and the mass conservation in each liquid phase :

Considering the axisymmetry of the laser-fluid interaction, we introduce cylindrical coordinates $(r, \theta, z)$ of the orthogonal basis $\left(\boldsymbol{e}_{r}, \boldsymbol{e}_{\theta}, \boldsymbol{e}_{z}\right)$. The $z$ axis is taken positive in the direction of propagation of the laser beam and the origin $z=0$ is set on the initially flat interface. The subscript $i=1,2$ denotes for the liquid phases 1 and 2 (see figure 2). The corrected pressure term $p_{i}$ contains the hydrostatic pressure and the gravitational component 


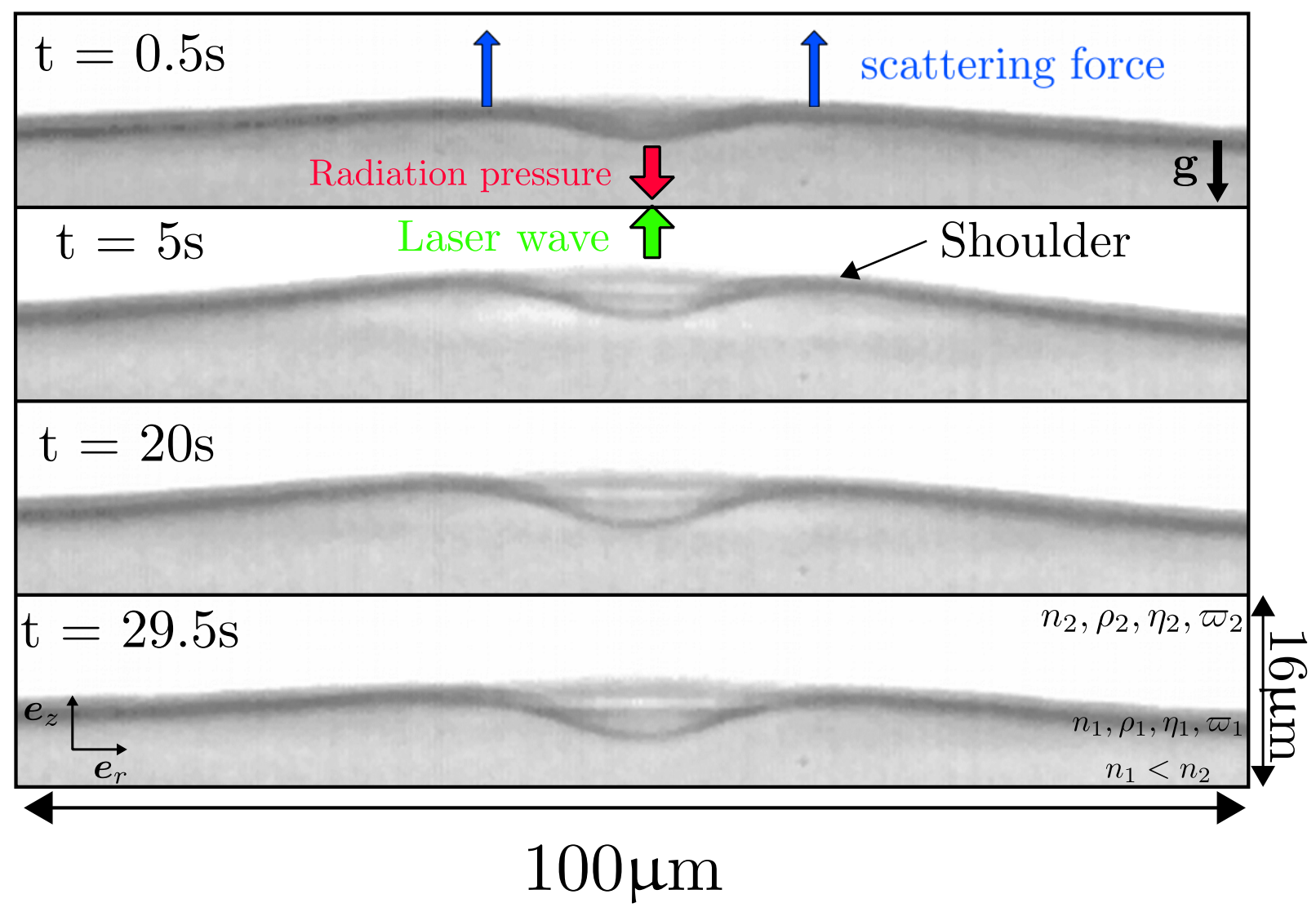

FIG. 2. Experimental evolution of the deformation of an initially flat interface under the mechanical effects of a laser beam. The laser is turn-on at $t=0 \mathrm{~s}$. This experiment was performed in a near-critical two-phase micro-emulsion at $T-T_{c}=1 \mathrm{~K}$, where $T_{c}$ is the critical temperature. The beam power is $P=308 \mathrm{~mW}$ and the beam waist is $\omega_{0}=9.8 \mu \mathrm{m}$. The green arrow denotes for the direction of propagation of the incident laser wave while the three others show where the manifestation of both the radiation pressure (red arrow from the large to the low refractive index phase, $n_{2}>n_{1}$ ) and the scattering force (blue arrows in the direction of propagation of the wave) induce visible effects on the fluid interface. The scattering deformation being wider than the radiation pressure one, the global deformation shows a circular shoulder indicated by the black arrow.

$p_{i}=p_{i}^{\prime}+\rho_{i} g h . \eta_{i}$ and $\boldsymbol{v}_{i}=v_{z i}(r, z) \boldsymbol{e}_{z}+v_{r i}(r, z) \boldsymbol{e}_{r}$ are respectively the viscosity and the velocity field of the fluid phase $i$ and $\boldsymbol{f}_{i}^{\text {scatt }}$ is the scattering force density. The detailed calculation of this force for single scattering from Rayleigh scatterers is given in appendix A. Equation (2) can be rewritten in a compact form as :

$$
\boldsymbol{f}_{i}^{s c a t t}=\varpi_{i} \frac{n_{i}}{c} I \boldsymbol{e}_{z}
$$

Where $\varpi_{i}$ is the turbidity coefficient and $n_{i} I / c$ is the light momentum in the liquid phase $i$. For the temperatures imposed experimentally, $\Delta T=T-T_{c}=0.5,1.5,1.9 \mathrm{~K}$, we respectively $\varpi_{1}=136 \mathrm{~m}^{-1}, 102 \mathrm{~m}^{-1}$ and $92 \mathrm{~m}^{-1}$. One can note also that $\varpi_{2} \approx \varpi_{1}$ close to a critical point. $I(r)=I_{0} \exp ^{-2\left(r / \omega_{0}\right)^{2}}$ is assumed as non-divergent during its propagation inside the whole sample. The stress balance at the interface is given by :

$$
\left[\mathbb{T}_{1}-\mathbb{T}_{2}\right] \cdot \boldsymbol{n}=[\sigma \kappa-\Delta \rho g h(r)] . \boldsymbol{n}
$$

With $\mathbb{T}_{i}=-p \mathbb{I}+\eta_{i}\left(\nabla \boldsymbol{v}_{i}+\nabla^{t} \boldsymbol{v}_{i}\right)$ the hydrodynamic stress tensor corrected by the pressure term and $\boldsymbol{n}$ a unit vector normal to the interface directed from fluid 1 to 2 . Finally, we impose continuity of the velocity at the interface, we use a Lagrangian description to track the interface motion and we consider a classical no-slip condition at the container boundaries.

$$
\begin{aligned}
& \frac{d \boldsymbol{x}}{d t}=\boldsymbol{v}(\boldsymbol{x}), \boldsymbol{v}_{1}=\boldsymbol{v}_{2}, \quad \text { at the interface. } \\
& \boldsymbol{v}_{1}=\boldsymbol{v}_{2}=\mathbf{0}, \quad \text { At the solid boundaries. }
\end{aligned}
$$


As we focus here on the dynamic effects of the scattering force over the interface, we did not include in our calculations a possible coupling with radiation pressure effects (already investigated numerically in stationary conditions and for small amplitude deformations [20]). Moreover, in order to focus on the effects of turbidity, we choose to consider $\eta_{1}=\eta_{2}$ in our simulations which is totally consistent with experiments in phase-separated near-critical mixtures as $\eta_{1} / \eta_{2} \in[1.07,1.11]$ for the range of temperature investigated here. We choose for the dimensions of the cylindrical numerical container of height $2 L$ and radius $R$ to consider $L=L_{1}=L_{2}=150 \omega_{0}$ for the liquid layer thicknesses and $R=60 \omega_{0}$ for their radial extension. These dimensions were chosen to discard as much as possible boundary effects due to finite volume of calculation. In addition, the radial extension was always chosen to be larger than $4 l_{c}$ for the range of investigated temperatures where $l_{c}$ is the capillary length $l_{c}=\sqrt{\sigma /(\Delta \rho g)}$. The length $4 l_{c}$ gives a confident distance from which one can consider the interface dynamic free of container sidewalls influence. We introduce two dimensionless numbers, an optical Bond number $B o=\left(\omega_{0} / l_{c}\right)^{2}=\left(\Delta \rho g \omega_{0}^{2}\right) / \sigma$ which describes the effects of buoyancy over capillarity with $\omega_{0}$ the beam waist as characteristic length (since we cannot discard radiation pressure experimentally), and a capillary number $C a_{i}=\eta_{i}\left(\partial v_{z i} / \partial z\right) /(\sigma / L)$ to represent the balance between viscous and interfacial stresses at the interface with $\mathrm{L}$ the characteristic length of the flow. As $v_{z i}$ depends on $\omega_{0}$ for $|r| \leq \omega_{0}$ (see figure 4 and 6 in [20]) we can deduce from equation (10) that $\eta_{i} \Delta v_{z i} \sim \eta_{i} v_{z i} / \omega_{0}^{2} \sim f_{i}^{\text {scatt }}$. Strictly speaking, it means that the capillary number $C a_{i}$ is space dependent due to the radial variation of the laser beam intensity and thus $C a_{i}=\left(2 n_{i} \varpi_{i}\right) /(\pi c \sigma) P e^{-2\left(\frac{r}{\omega_{0}}\right)^{2}}$. Conversely, due to the inherent radiation pressure surface effects (not considered for the sake of simplification in the simulations) on the beam axis of the experiments that opposes to scattering interface deformations, the largest observable manifestation of scattering flows appears at the shoulder $r \approx \omega_{0}$ (see figure 2 for maximum hump amplitude and figure 4 for the jetting instability). Thus the experimental capillary number should be evaluated at $r \approx \omega_{0}\left(\right.$ as $\left.\Pi_{r a d}\left(r=\omega_{0}\right) \approx 0\right)$ to get quantitative comparisons between experimental and numerical results ; it is then given by $C a_{i}=\left(2 n_{i} \varpi_{i}\right) /(\pi c \sigma) P / e^{2}$. Additionally, as experimentally the jet is formed on the shoulder of the deformation, i.e. where the scattering force contribution is the largest in presence of radiation pressure, we would like to compare the viscous stress and the Laplace pressure at this location. However, as it is not obvious to determine exactly the initial position of the jet nor the local viscous stress in situ, we decided to compute the experimental capillary number by introducing an effective power taking into account the radial decrease of the intensity. Indeed, as the jet is misaligned with the beam axis, defining a "centered" capillary number leads to irrelevant experimental thresholds capillary numbers $\left(C a_{t h} \in[5-10][21]\right)$.

In the next section, we describe the dynamics of the deformed interface by scattering forces before the jetting instability threshold. We made the choice hereafter to present all capillary numbers using $C a_{1}$ as one should note that close to a critical point, where scattering effects are the largest, $C a_{2} \approx C a_{1} \equiv C a$.

\section{BELOW THRESHOLD}

It was previously demonstrated that the scattering force density can produce small deformations [20] of a soft liquid/liquid interface by inducing bulk flows in turbid media in the form of toroidal eddies. Typical pictures of the dynamics of a small amplitude deformation is depicted figure 2. We report on figure 3 the experimental (open symbols) and the numerical (dashed lines) evolution of the shoulder height normalized by the beam waist with the viscocapillary time defined with the beam waist as length scale $\tau=\left(\omega_{0} \eta_{2}\right) / \sigma$ (the viscous reference velocity of the interface is $U_{\sigma}=\omega_{0} / \tau$ ) for different Bond numbers (including beam waist variation) and for several capillary numbers (including beam power variation). The experimental capillary numbers are calculated as discussed in the previous section and the corresponding injected powers are given in the figure caption. Two regimes are noticeable on the experimental dynamics. First, at short time, the interface is deformed by the viscous stress induced by the scattering force in the direction of propagation of the laser beam. Then, at longer time, the interface is pulled back in the opposite direction by radiation pressure. The numerical dynamics in 2 , which consider only scattering force, show clearly that the radiation pressure is involved in the long-time process as the numerical deformations simply grow and reach a stationary state. As the time scales observed experimentally are well separated, we propose an empirical law which considers the addition of the deformation induced by the scattering force and the pull back due to radiation pressure :

$$
h(t)=\alpha\left[\left(1-e^{\left(-\frac{t}{\tau_{1}}\right)}-\beta\left(1-e^{\left(-\frac{t}{\tau_{2}}\right)}\right)\right]\right.
$$

With $\alpha, \beta, \tau_{1}$ and $\tau_{2}$ four fitting parameters. The empirical law (plain lines in figure 3) describes well the experimental measurements. We obtain two characteristic times, $\tau_{1}$ which corresponds to the effect of the scattering force at short time scale and $\tau_{2}$ assigned to the second slower phenomenon related to radiation pressure. For all the dynamics we find $\tau_{1} \in[0.45,0.65] s$ which scales like $L_{c} / v^{\text {scatt }}[20]$, with $L_{c}$, the smallest dimension of the container and $v^{\text {scatt }}=\left(f_{i}^{\text {scatt }} \pi \omega_{0}^{2}\right) / \eta_{i}=\left(2 P n_{i} \varpi_{i}\right) /\left(c \eta_{i}\right)$ derived from a one-fluid model. The fact that $\tau_{1}$ is independent of the 

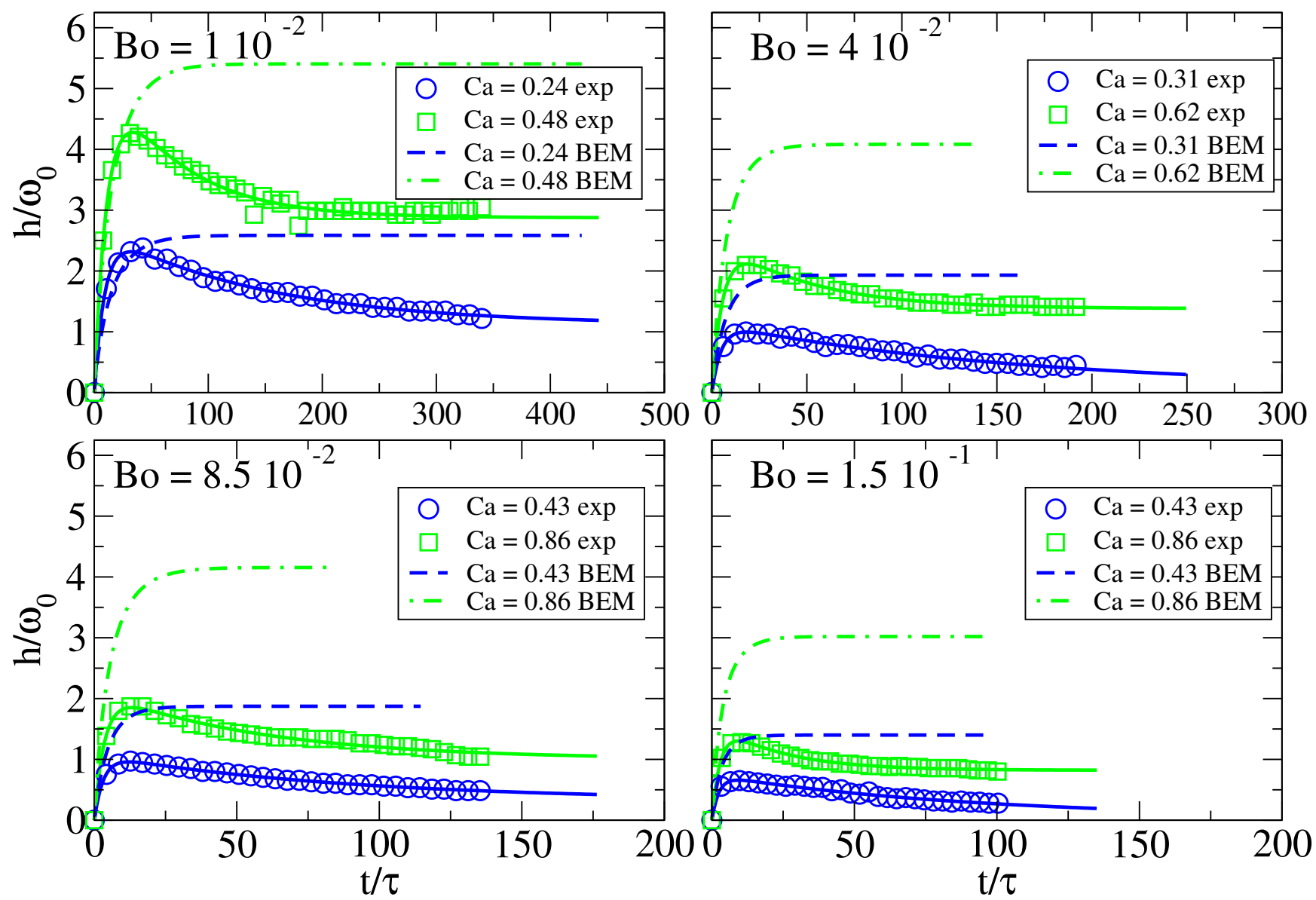

FIG. 3. Dynamics of the interface shoulder at different Bond numbers (with, from top-left corner to bottom-right one, $\omega_{0}=$ 2.7, 5.3, 7.5, $9.8 \mu \mathrm{m}$ ) and for several capillary numbers (with $\mathrm{P}=346.5 \mathrm{~mW}, 693 \mathrm{~mW} ; \mathrm{P}=442 \mathrm{~mW}, 885.5 \mathrm{~mW} ; \mathrm{P}=616 \mathrm{~mW}$, $1232 \mathrm{~mW} ; 616 \mathrm{~mW}, 1232 \mathrm{~mW}$ from top-left to bottom-right). Since radiation pressure is also present, the measurements are taken on the shoulder of the deformation (see figure 1c), where the scattering force effects are the most visible. All these results were obtained at $T-T_{c}=1.5 \mathrm{~K}$. Open symbols are experimental data and are fitted (plain lines) with equation (14). Dashed lines are numerical simulations considering scattering forces only.

beam waist $\omega_{0}$ expresses again the non-local feature of the interface deformation induced by the scattering force. Additionally, the time scale associated to the numerical dynamics $\tau_{n u m} \in[0.7,0.9] \mathrm{s}$ is close to the experimental one and shows that $\tau_{1}$ is associated to the scattering force. Conversely, $\tau_{2}$ ranges from a few to tens of seconds. It has been demonstrated in previous studies that deformations induced by significant radiation pressure act as liquid waveguides [33] with complex adaptation of the incident spatial field distribution. A given amount of eigenmodes of the field is guided into the deformation which modifies in turn the radiation pressure. Depending on the beam power and waist, this modified field may pull the central deformation from an equilibrium $z$-position to another. Reaching a new stationary state may take time as the field and the interface continuously adapt to each other. This complex nonlinear behavior of the radiation pressure [33] can explain the decreasing of the height amplitude observed at long time in figure 3.

In the following, we will see that when we increase sufficiently the capillary number above a threshold value denoted $C a_{t h}$, the scattering contribution becomes large enough to destabilize the interface. A "fountain" jet emerges on the shoulder of the deformed interface as illustrated in figure 4. Statistically, this jet can form in any place on the circular shoulder. However, in the experiments the jetting breaks the symmetry, and therefore it becomes impossible to directly simulate the real system because of the axisymmetry of our simulation. As introduced before, (i) we discard radiation pressure effects which do not produce stationary flow, (ii) we choose to keep simulating liquid jets centered on the propagation axis of the laser beam and (iii) we compare numerical results with experimental ones considering their respective capillary numbers. 

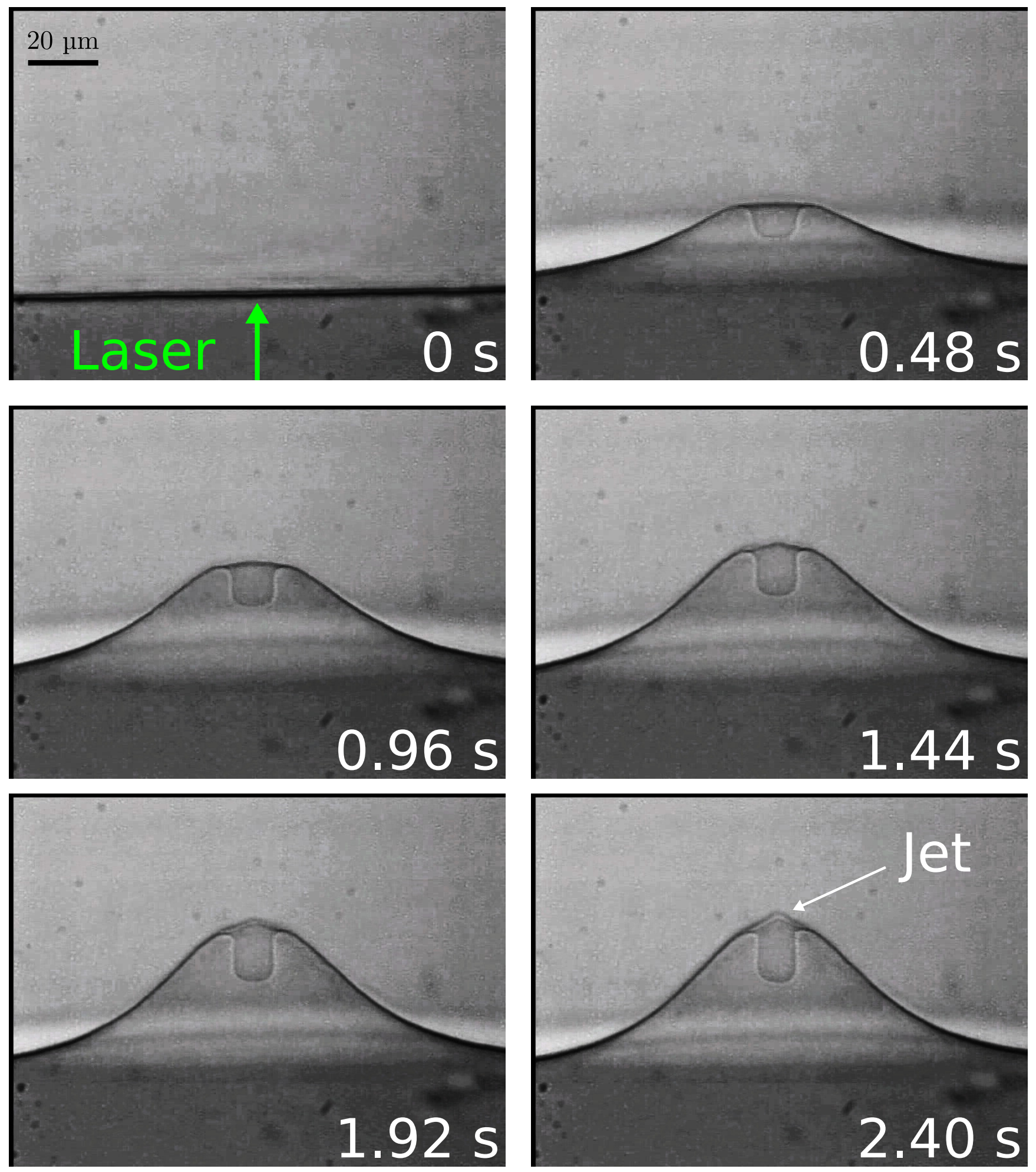

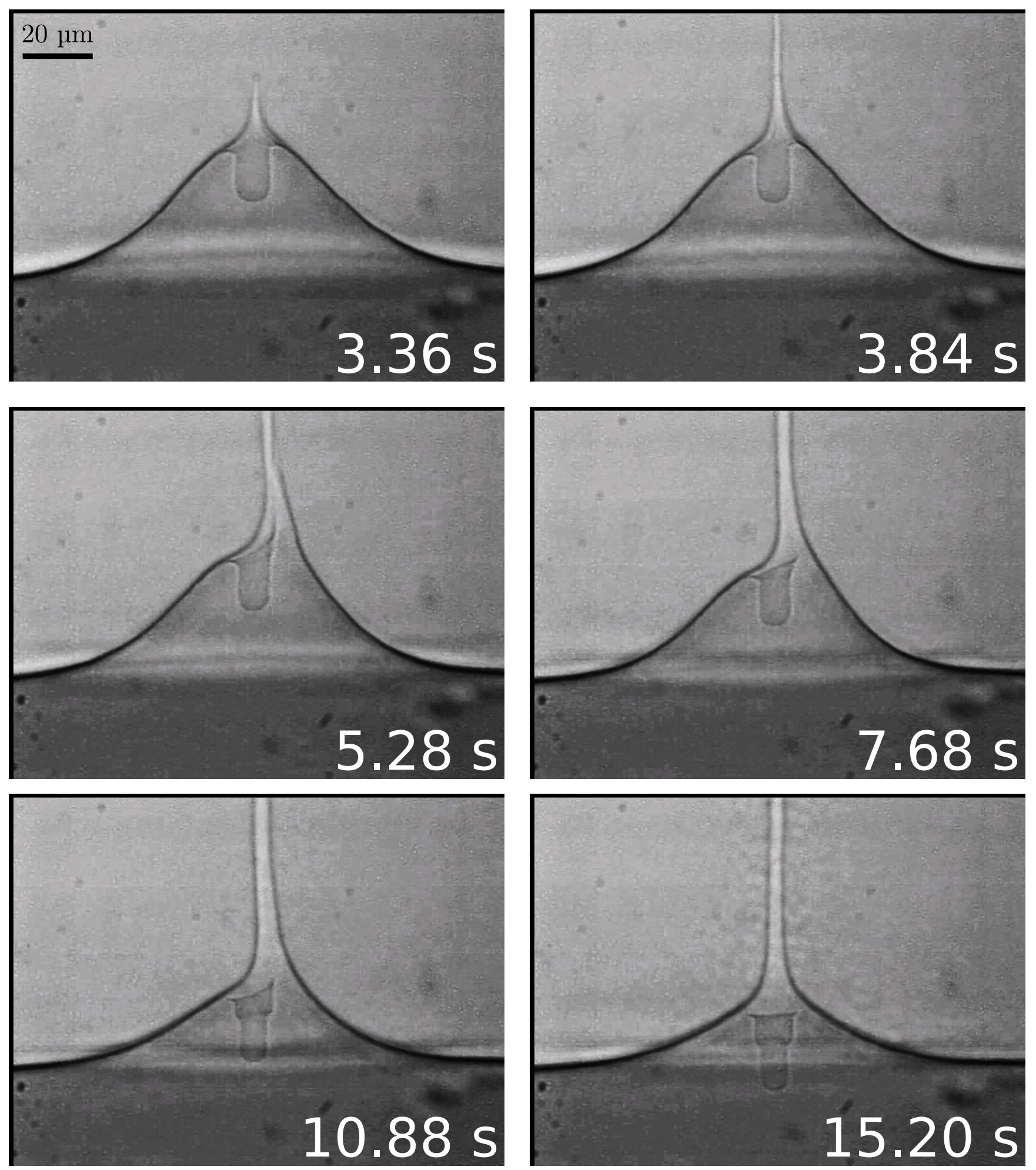

FIG. 4. Temporal evolution of the deformation of the soft liquid interface followed by its destabilization and emergence of a micro-jet. $T-T_{c}=1.5 \mathrm{~K}, P=1540 \mathrm{~mW}$ and $B o=8.6 \times 10^{-2}$ with $\omega_{0}=7.5 \mu \mathrm{m}$. At time $t=0 \mathrm{~s}$ the laser is turned on. The white arrow highlights the emergence of a liquid tip at the shoulder of the deformation which turns into a micro-jet in the wave propagation direction ; note that this jet is shifted regarding to the beam axis due to the fact that it is constituted by the liquid phase of smallest index of refraction. It seems to be centered at the end of the movies, but it had just moved in front of the camera; the shoulder is still present. 


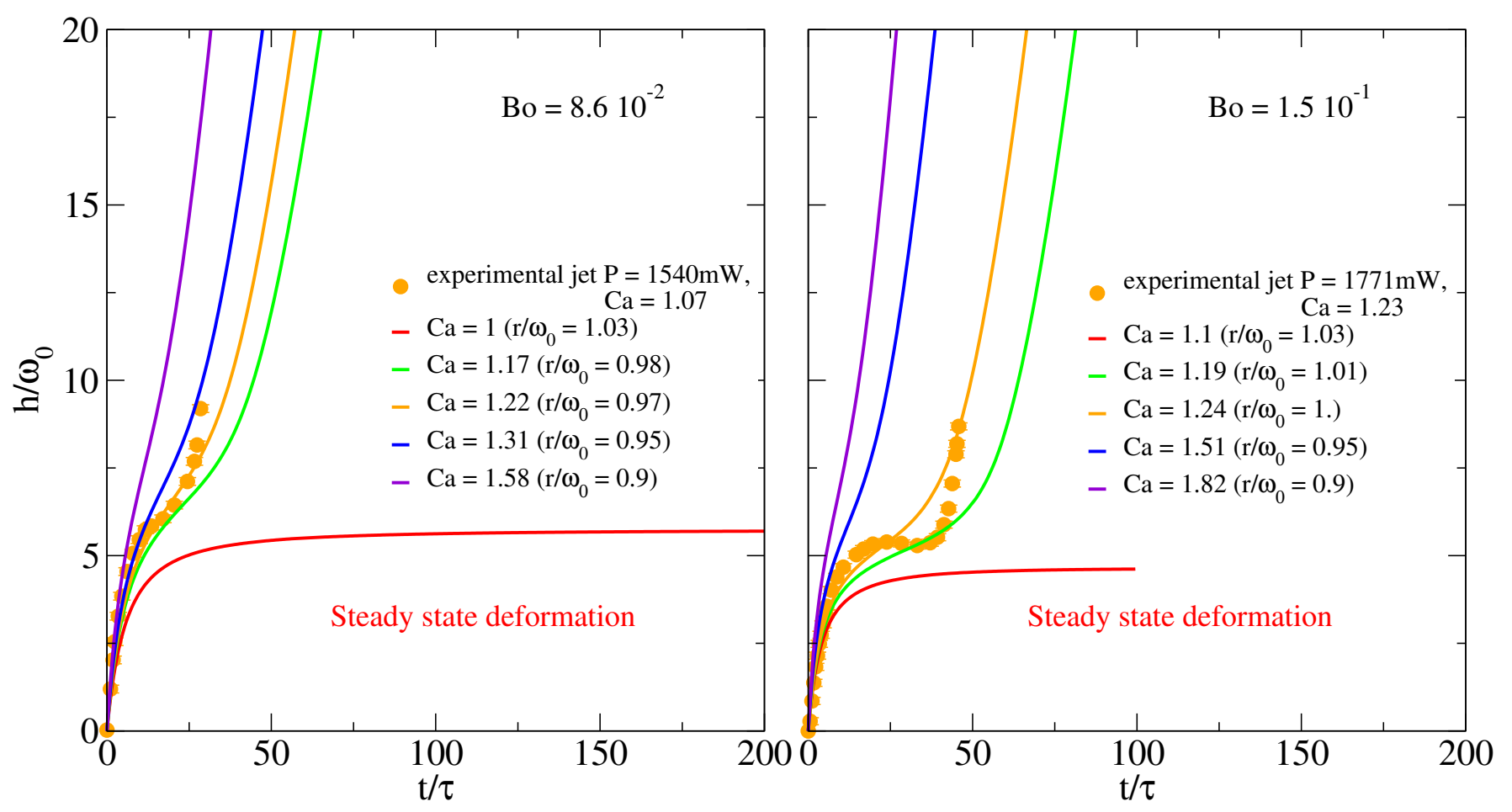

FIG. 5. Comparison between the height evolution of experimental jets (filled dots) and numerical ones (lines). Both experimental jets were obtained at $T-T_{c}=1.5 \mathrm{~K}$ and the Bond number are calculated with $\omega_{0}=7.5,9.8 \mu \mathrm{m}$. The experimental dynamics (see figure 4) are considered at short times on the shoulder of the deformation and then at the apex of the jet. Experimentally, we do not observe jets larger than $h / \omega_{0}>10$ due to the limited size of the camera chip. Numerically the height of the interface is taken at $r=0$ as we discarded radiation pressure effects.

\section{BEYOND THRESHOLD}

As a validation of our approach, we compare the dynamics of experimental and numerical jets. We plot in figure 5 the evolution of experimental and numerical deformations actuated by the scattering force using the viscocapillary time. We then compare experimental dynamics with several predicted ones for different capillary numbers. Following the discussion in the section II and considering a spatially dependent capillary number, the computed numerical dynamics correspond to different radial positions where the jet would be induced experimentally. As expected the best match is retrieved in figure 5 by considering a shoulder at $\frac{r}{\omega_{0}} \approx 1$, which is in agreement with the experimental jet position. Then, in order to get experimental capillary numbers comparable with numerical ones, we choose in the following to always consider $C a_{\text {exp }}$ at $r / \omega_{0}=1$.

In the next sections, we describe the different properties of the induced experimental and numerical liquid microjets. We compare the velocity field inside the jets computed numerically with an analytical model, characterize their radii and finally determine the resulting fluid flow rates.

\section{A. Velocity}

In order to calculate the fluid flow rate through the numerical jets, we computed the bulk velocity field by BEM.

We observe on figure 6 an experimental jet with a corresponding numerical one superimposed on the same image (panel (a)) and the same numerical jet plotted along with the streamlines of the induced velocity field (panel (b)) obtained with a capillary number $\mathrm{Ca}=1.08$. Disregarding the fact that the experimental jet is misaligned with the laser beam, both jets are very similar and extremely thin (few micrometers in diameter).

As expected from mass conservation, we observe the formation of toroidal flows which scale as the smallest dimension of the container (here the radial extension) in each liquid phase. The same kinds of flows can be observed experimentally (see figure 1 of [20]). Note that by linearity of the Stokes equation (10) the velocity is proportional to $f^{\text {scatt }}$ and thus to $C a$. To validate the computed velocity field, we also calculated analytically the hydrodynamic velocity we would obtain considering a perfect cylindrical jet [19]. We consider the flow into the jet as a viscous 
(a)

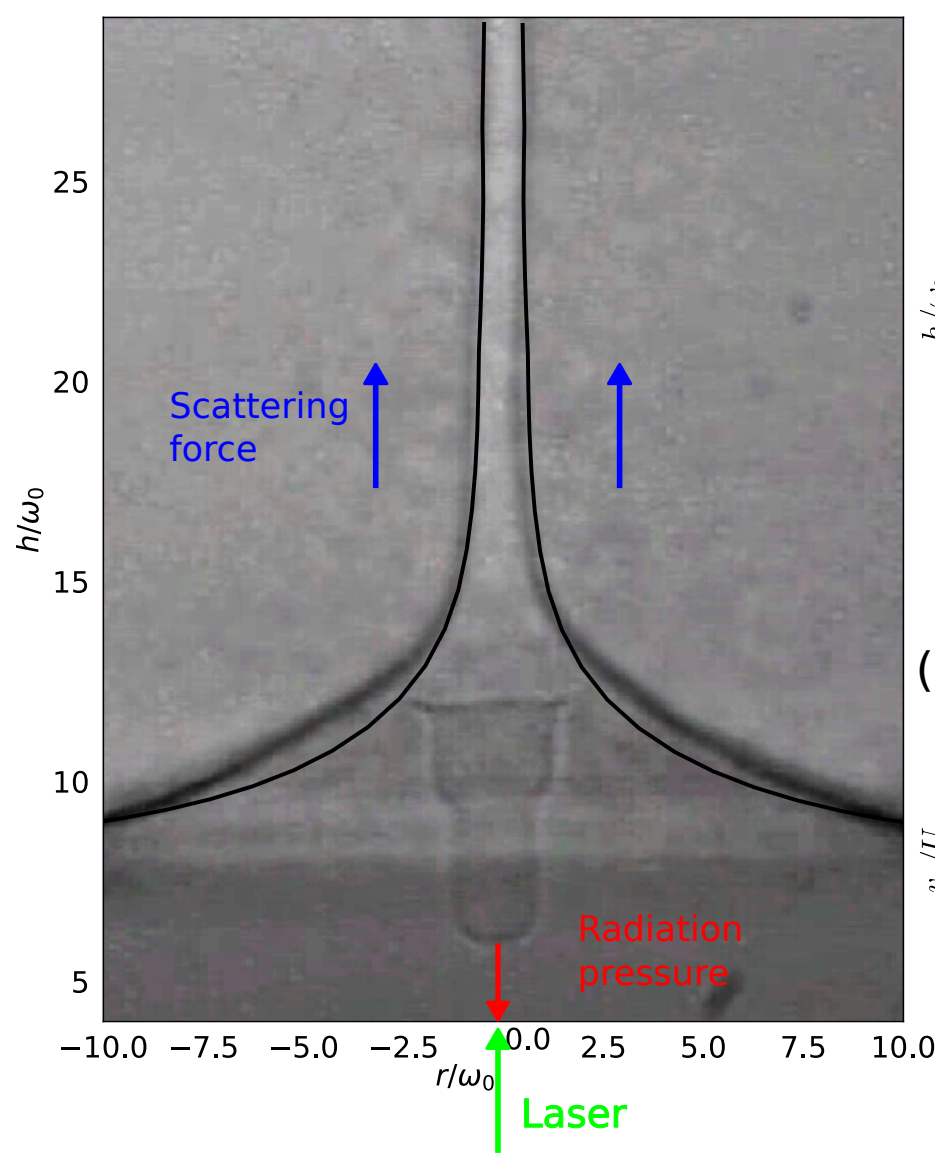

(b)
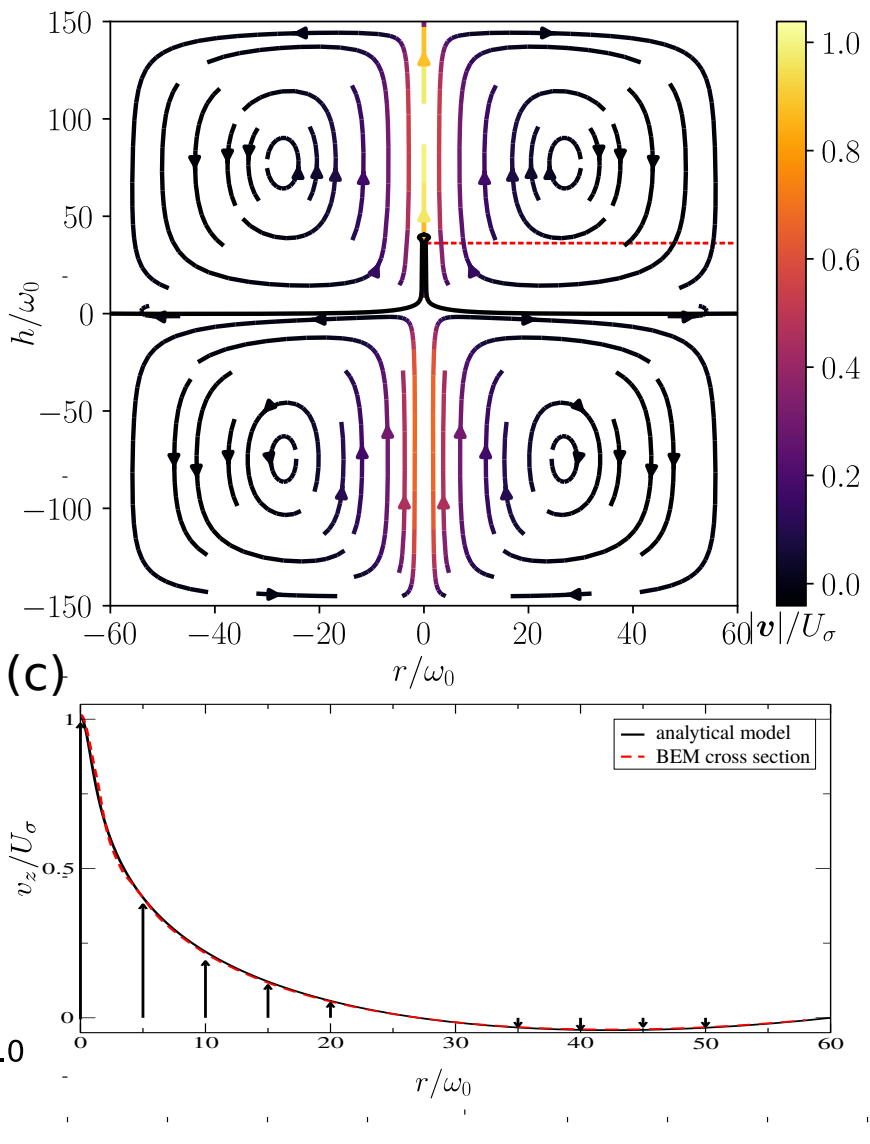

FIG. 6. Analysis of the hydrodynamic velocity field. (a) Experimental image of a jet induced by the scattering force and the corresponding simulated profile at $T-T_{c}=1.5 \mathrm{~K}, B o=8.6 \times 10^{-2}\left(\omega_{0}=7.5 \mu \mathrm{m}\right), C a=1.07(P=1540 \mathrm{~mW})$. (b) Numerical flow pattern of the velocity field at $B o=8.6 \times 10^{-2}$ and $C a=1.07$. (c) Comparison between the velocity field at the cross-section represented by the dashed red line in (b) with the analytical solution obtained for a perfect cylindrical jet.

incompressible steady laminar flow for which the velocity field has only radial dependences along the z axis. Furthermore, as the scattering force also depends on $r$ along $z$, equation (10) implies $\partial_{r} p_{i}=0$ which tells us that the corrected pressure only depends on z. Then by taking the divergence of equation (10), one finds $\partial_{z}^{2} p_{i}=0$. So the pressure gradient is constant in both liquid phases $\partial_{z} p_{i}=C_{i}$.

To obtain the whole expression of the pressure, we use equation (12). For $r \gg \omega_{0}$, and at $z=0$, the interface is flat (curvature is null) and the amplitude of the hydrodynamic velocity field is negligible (see figure 6 panels (b)), so equation (12) gives us $p_{1}(z=0)=p_{2}(z=0)=p_{0}$ the pressure at $\mathrm{z}=0$. Finally, as all terms of equation (12) are invariant along $z$ axis, $p_{1}(z)-p_{2}(z)$ should also be constant. Hence, we must choose $C_{1}=C_{2}=C$. We can now rewrite equation (10) in a dimensionless form with the characteristic lengths chosen earlier :

$$
\frac{1}{r} \frac{d}{d r}\left(r \frac{d}{d r} v_{z i}(r)\right)=C-\frac{C a_{i}}{\alpha_{i}} e^{-2 r^{2}}
$$

$$
\alpha_{i}=\left\{\begin{array}{lll}
\frac{\eta_{2}}{\eta_{1}} & \text { if } & i=1 \\
1 & \text { if } & i=2
\end{array}\right.
$$

By integrating these equations in each liquid phase, we get the following coupled equations :

$$
v_{z 1}(r)=\frac{C}{4}\left(r^{2}-r_{1}^{2}\right)+\frac{C a_{1}}{4 \alpha}\left(\frac{E_{i}\left(-2 r^{2}\right)}{2}-\frac{E_{i}\left(-2 r_{1}^{2}\right)}{2}-\ln \left(\frac{r}{r_{1}}\right)\right)
$$




$$
\begin{aligned}
v_{z 2}(r)= & \frac{C}{2}\left\{\frac{1}{2}\left(r^{2}-r_{3}^{2}\right)-r_{2}^{2} \ln \left(\frac{r}{r_{3}}\right)\right\}+\frac{C a_{2}}{4}\left(\frac{E_{i}\left(-2 r^{2}\right)}{2}-\frac{E_{i}\left(-2 r_{3}^{2}\right)}{2}\right. \\
& \left.-e^{-2 r_{2}^{2}} \ln \left(\frac{r}{r_{3}}\right)\right)
\end{aligned}
$$

with $E_{i}$ the exponential-integral function and $C, r_{1}, r_{2}$, and $r_{3}$ four unknowns which are calculated numerically by considering (i) the continuity of the tangential velocity at the interface, (ii) the continuity of the shear stress at the interface, (iii) a no-slip condition on the domain boundaries and (iv) a flow rate outside the jet (into the liquid phase 2) equal to zero due to mass conservation. We plot in figure 6 panel (c), the comparison between the numerically calculated velocity over a cross-section of the jet and the corresponding analytical solution. These velocity profiles match perfectly.

\section{B. Radius}

We need now to characterize the jet radii. Experimentally and numerically, as the jets are never perfectly cylindrical, the reported radii are taken as the mean ones of the most cylindrical part of the jets. Thus, we plot in figure 7 the variation of the jet radii with the capillary number ; the insets show (a) their variation from the threshold of jet formation in a logarithmic scale with $R_{t h}$ and $C a_{t h}$ respectively the radius and the capillary number at the threshold and (b) the viscous stress on the jet interface. We retrieve a good agreement between numerical and experimental results despite some dispersion into the measurements of the experimental radii and a small systematic overestimation of the numerical jet radius likely due to the asymmetric character of the experimental configuration (see figure 4). Furthermore, figure 7 inset (a) shows that the jet radius, relatively to its threshold value, do not depend on the Bond number, and therefore demonstrates again the nonlocal nature of the scattering force. Simple scaling arguments can be given to explain this linear behavior. Indeed, we assume that the radial viscous stress on the jet interface should have a negligible variation so that $\partial v_{z} / \partial r \sim A$ with $A$ a constant. By definition of the capillary number on a cylindrical jet with small interface fluctuations, we have $C a=\eta\left(\partial v_{z i} / \partial r\right) /\left(\sigma / R_{j e t}\right)$ and therefore $C a \sim A \times R_{\text {jet }}$. Finally, increasing the beam power, i.e. $C a$, will lead to a proportional increase of $R_{\text {jet }}$ in order to avoid any subsequent increase of the viscous stress which is maintained constant at the interface. This is demonstrated numerically in figure 7 inset (b) where we plotted the viscous stress normalized by the viscosity on the jet interface as a function of its radius ; we observe that $\partial v_{z} / \partial r$ is constant for $R_{\text {jet }} / \omega_{0} \geq 1$.

Once simulated jet radius and flow are characterized, we calculate next the fluid flow rates and compare them to the experimental ones.

\section{Fluid flow rate}

Experimentally the fluid flow rate is calculated by measuring the volume of the drops emitted at the tip of the micro-jet during several seconds. The total volume ejected is then divided by the whole measurement time to obtain the corresponding mean flow rate [19], see the snapshot on figure 8. To determine the numerical fluid flow rate, we integrate the computed fluid velocity over a cross-section of the cylindrical jet :

$$
Q=2 \pi \int_{0}^{R_{j e t}} v_{z 1} r d r
$$

As $R_{\text {jet }} \propto C a$ and $v_{z} \propto C a$ we should expect the fluid flow rate to be proportional to $C a^{3}$. This cubic dependence of the fluid flow rates with the capillary number is retrieved numerically and the same order of magnitude is observed when comparing with the experiments ; thus qualitative agreement is illustrated in figure 8(a) and (b). However, discrepancies in amplitude between experimental and numerical fluid flow rates are observed. They probably come from the fact that experimentally we determine the fluid flow rates by considering the emitted volume of fluid at the tip of the jet and not inside a jet cross section. On the one hand, the break-up at the jet tip and then the emission of droplets are affected by the Laplace pressure through the interfacial tension. On the other hand, when a droplet is ejected, the jet undergoes a recoil which can affect the hydrodynamics in a more complex way than the one considered in this article. Moreover, these discrepancies can also come from several other factors. For example, the chosen dimensions in the simulations allow us to discard any boundary effect but it may not always be true in the experiments. Indeed, due to the jet length, the droplets are ejected close to the top wall of the cell where the axial velocity decreases quickly. Additionally, if the laser is focused on the interface close to the radial boundaries (which is 


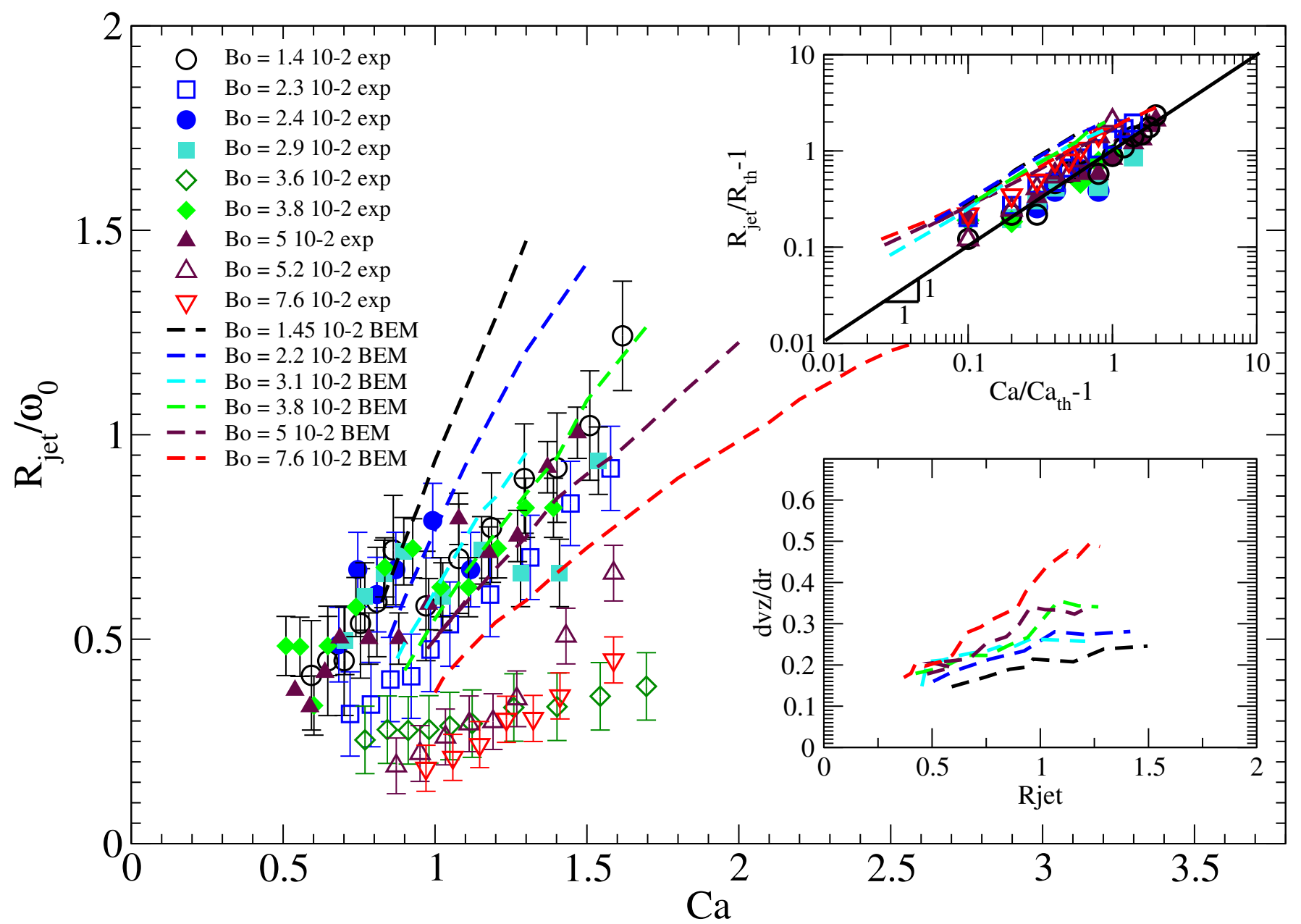

FIG. 7. Variation of the micro-jet radius with the capillary number for different Bond numbers ; corresponding to the experimental beam waists $\omega_{0}=4.4,4.8,5.5,6.4,3.0,3.9,4.4,5.9,7.1 \mu \mathrm{m}$. Numerical jet radii are represented by dashed lines and experimental ones with open $\left(T-T_{c}=1.5 \mathrm{~K}\right)$ and filled $\left(T-T_{c}=1.9 \mathrm{~K}\right)$ symbols. Inset (a) : Same data normalized by their value at the jetting threshold $R_{t h}$ and $C a_{t h}$ in $\log -\log$ plot. Inset (b) : Evolution of the numerical viscous stress at the jet interface, normalized by the viscosity, with the jet radius for the Bo numbers considered numerically (same color code).

the case in the experiments in order to improve optical contrast) it may also affect the hydrodynamic by reducing the fluid flow rates. Nonetheless, it was found in reference [19], with a different configuration (when the resulting stress of the radiation pressure and the scattering force are both collaborating in the same direction), that the experimental fluid flow rate increases as $\mathrm{Ca}^{2.63}$, which is close to our numerical results ; in this collaborating configuration, the jet is much more stable as the laser beam propagates into it and stabilizes it by mode guiding.

In further works it could be interesting to try to simulate dripping jets to characterize numerically the fluid flow rate through ejected droplets, in order to have a better understanding of the present discrepancies.

\section{CONCLUSION}

We described experimentally and numerically the whole dynamics of deformations of soft interfaces by light scattering density forces up to instability and the emergence of liquid fountains. We first analyzed the dynamics of interface deformations below instability threshold and measured the associated characteristic times. Using an empirical expression which fits well with the whole set of experimental dynamics, we showed that deformations are initially induced by a non-local effect which corresponds to the viscous stress exerted by the bulk flow triggered by the momentum transfer of light in turbid liquids. In a second part, increasing the amplitude of the bulk flows driven by light scattering, we described the interface behavior above its instability threshold at which liquid micro-jets emerge. We characterized their radius evolution and demonstrated that they increase linearly with the capillary number. By computing the 

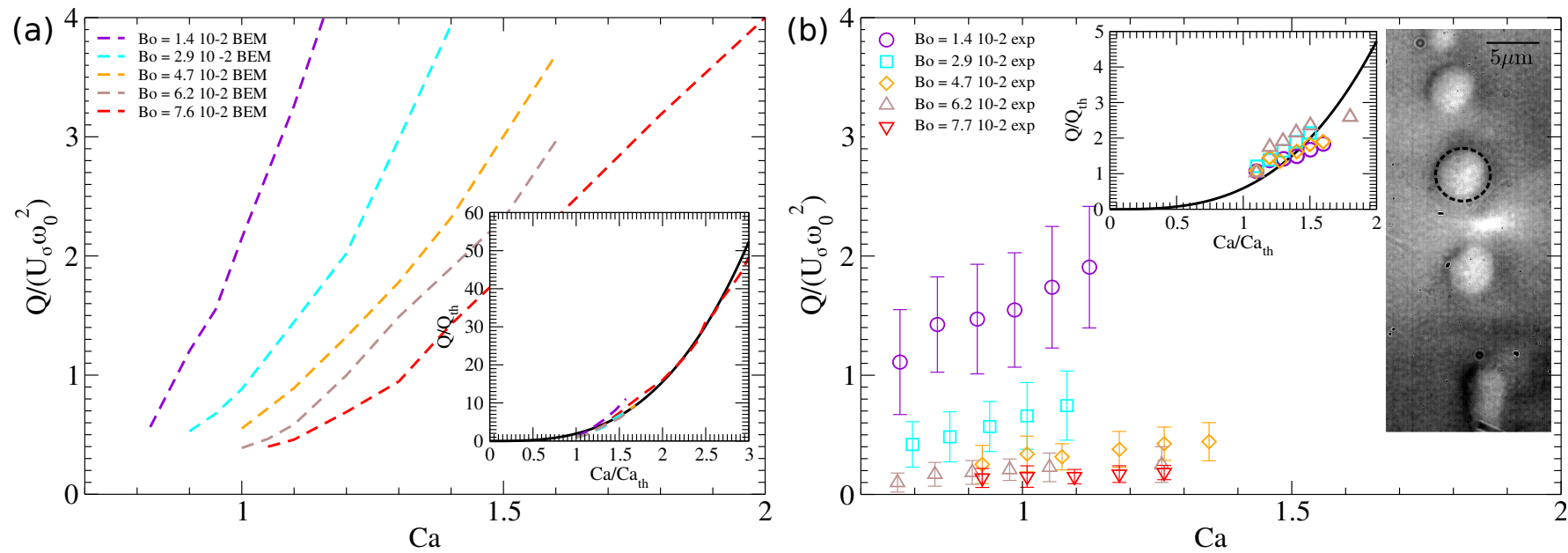

FIG. 8. (a) Variation of the numerical (dashed lines) and (b) experimental (open symbols) fluid flow rates with the capillary number for different Bond numbers. The insets show the same data normalized by their value at the threshold. The plain black line in both insets is a fit of the whole set of curves according to $Q / Q_{t h}=a_{0} \times\left(C a / C a_{t h}\right)^{3}$ with $a_{0}=1.87$ for BEM results and $a_{0}=0.59$ for experimental ones. All the experimental results plotted here were obtained at $T-T_{c}=1.5 \mathrm{~K}$ and their Bond numbers are calculated with $\omega_{0}=3.0,4.4,5.5,6.4,7.1 \mu \mathrm{m}$. The snapshot shows an example of droplet train emitted at the tip of a jet, and how they are fitted with an ellipse to calculate their volume.

hydrodynamic velocity field flowing through the jets, we were able to determine the numerical fluid flow rate which increases as the cube of the capillary number and compare it to the experimental ones.

All the results presented here bring important new insights in the area of light-actuation of fluids in general and describe a unique way for triggering jets by light at low Reynolds number. Even if our experiments were performed in near-critical fluids in order to control the medium turbidity and then the amplitude of the scattering force, scattering forces effects can be generalized to any type of non-absorbing suspension. In reference [34] the authors already shown probable manifestations in colloidal solutions and we already demonstrated scattering effects in so-called turbid L3 phases [21]. Consequently, scattering forces through momentum transfer from photons to turbid fluids proves to be an efficient way for setting fluid suspensions in movement in the micro-world without using any sort of pump or mechanical parts and the present investigation quantify the capabilities of such a contactless optical actuation.

\section{ACKNOWLEDGMENTS}

The authors thank Thomas Sidky for early-stage experiments on flow rate measurements.

\section{Appendix A: Scattering effects in turbid liquids}

In this section we present the calculation of the scattering force due to the interactions of an incident laser wave with a turbid fluid. Let us consider a dielectric and isotropic fluid, with a mean dielectric constant $\langle\varepsilon(\boldsymbol{r}, t)\rangle=\varepsilon_{l}=\varepsilon_{0} \varepsilon_{r}$, in which an incident plane wave is propagating within the form $\boldsymbol{E}_{i}(\boldsymbol{r}, t)=\boldsymbol{n}_{i} E_{0} \exp \left[i\left(\boldsymbol{k}_{i} \cdot \boldsymbol{r}-\omega_{i} t\right)\right]$, of polarization $\boldsymbol{n}_{i}$, of frequency $\omega_{i}$ and of wave vector $\boldsymbol{k}_{i}=\left(\omega_{i} / c\right) \widehat{\boldsymbol{k}}_{i}$. The fluid is assumed as turbid meaning that the local dielectric constant includes a fluctuating part such as: $\varepsilon(\boldsymbol{r}, t)=\varepsilon_{l}+\delta \varepsilon(\boldsymbol{r}, t)$. Furthermore, the dielectric medium is considered as non-absorbing so that the interaction of the incident wave with the scatterers is elastic and the number of scatterers is considered as sufficiently low to neglect multiple scattering events.

We consider that the amplitude of the field scattered by the fluctuations $\delta \varepsilon$ is significantly weaker than the incident field. The scattered field as well as the incident field satisfying the Maxwell equations, one can obtain the expression 
of the scattered field in the Born approximation [35]

$$
\boldsymbol{E}_{S}(R, t)=\frac{E_{0}}{4 \pi \varepsilon_{l} R} \exp \left[i\left(k_{f} R-\omega_{i} t\right)\right] \boldsymbol{k}_{f}\left[\boldsymbol{k}_{f} \times \boldsymbol{n}_{i} \int_{V} \mathrm{~d}^{3} r \exp [\mathrm{i}(\mathrm{q} \cdot \mathrm{r})] \delta \varepsilon(\mathrm{r}, \mathrm{t})\right],
$$

where $R$ is the distance between the turbid medium and the detector, $\boldsymbol{k}_{f}$ the wave vector in the direction of $\boldsymbol{R}$ and $\boldsymbol{q}=\boldsymbol{k}_{i}-\boldsymbol{k}$. Considering geometric arguments, one can rewrite equation (A1) as:

$$
\boldsymbol{E}_{S}(R, t)=\frac{k_{f}^{2} E_{0}}{4 \pi \varepsilon_{l} R} \exp \left[i\left(k_{f} R-\omega_{i} t\right)\right] \delta \varepsilon(\boldsymbol{q}, t)\left(\cos \varphi \widehat{k}_{f}-\boldsymbol{n}_{i}\right),
$$

where $\varphi$ represents the angle between the polarization of the incident wave $\boldsymbol{n}_{i}$ and the scattered wave vector direction $\widehat{k}_{f}$. The scattering intensity is related to the auto-correlation function of the scattering field $\boldsymbol{E}_{S}$, such as:

$$
\left\langle\boldsymbol{E}_{S}^{*}(R, 0) \cdot \boldsymbol{E}_{S}(R, t)\right\rangle=\frac{k_{f}^{4}\left|E_{0}\right|^{2}}{16 \pi^{2} \varepsilon_{l}^{2} R^{2}}\langle\delta \varepsilon(\boldsymbol{q}, 0) \delta \varepsilon(\boldsymbol{q}, t)\rangle \exp \left(-i \omega_{i} t\right)\left|\cos \varphi \widehat{k}_{f}-\boldsymbol{n}_{i}\right|^{2}
$$

where $\langle A\rangle=\lim _{T \rightarrow \infty}(1 / T) \int_{0}^{T} A(t) d t$. Thus, the spectral density of the scattered light measured by a detector placed at a distance $R$ is given by:

$$
I\left(\boldsymbol{q}, \omega_{f}, R\right) \equiv \frac{1}{2 \pi} \int_{-\infty}^{+\infty} d t \exp \left(i \omega_{f} t\right)\left\langle\boldsymbol{E}_{S}^{*}(R, 0) \cdot \boldsymbol{E}_{S}(R, t)\right\rangle .
$$

After some developments, one can write the total scattering intensity as :

$$
I\left(\boldsymbol{q}, \omega_{f}, R\right)=\left[\frac{I_{0} k_{f}^{4} \sin ^{2} \varphi}{16 \pi^{2} \varepsilon_{l}^{2} R^{2}}\right]\left\langle|\delta \varepsilon(\boldsymbol{q})|^{2}\right\rangle,
$$

with $I_{0}=\left|E_{0}\right|^{2}$ the intensity of the incident wave and $\left\langle|\delta \varepsilon(\boldsymbol{q})|^{2}\right\rangle$ representing the scattering properties of the turbid medium. After the evaluation of the term $\left\langle|\delta \varepsilon(\boldsymbol{q})|^{2}\right\rangle$ in equation (A5) in the Ornstein-Zernike approximation [35] for quasi-critical fluids, one comes up with :

$$
I\left(\boldsymbol{q}, \omega_{f}, R\right)=\left[\frac{I_{0} k_{f}^{4} \sin ^{2} \varphi}{16 \pi^{2} \varepsilon_{l}^{2} R^{2}}\right]\left|\frac{\partial \varepsilon_{l}}{\partial \rho}\right|^{2} \beta^{-1} \rho \chi_{T}\langle N\rangle\left[\frac{1}{1+\left(\frac{q}{q_{0}}\right)^{2}}\right],
$$

where $\beta=1 /\left(k_{B} T\right), \chi_{T}$ is the isotherm compressibility and $q_{0}^{-1}=\xi$ the correlation length of density fluctuations. As $\rho=\langle N\rangle / V$, with $\langle N\rangle$ the average number of scatterers in volume $V$ of the scattering medium, $\varepsilon_{l}=\varepsilon_{0} \varepsilon_{r}$ and $k_{f}=\left(2 \pi \sqrt{\varepsilon_{r}}\right) / \lambda_{0}=(2 \pi n) / \lambda_{0}\left(\lambda_{0}\right.$ being the wavelength of the incident beam in vacuum), we can write :

$$
\frac{I\left(\boldsymbol{q}, \omega_{f}, R\right)}{I_{0}}=\frac{\pi^{2}}{\lambda_{0}^{4}}\left(\rho \frac{\partial \varepsilon_{r}}{\partial \rho}\right)^{2} k_{B} T \chi_{T}\left[\frac{1}{1+\left(\frac{q}{q_{0}}\right)^{2}}\right] \frac{V}{R^{2}} \sin ^{2} \varphi .
$$

In the equation (A7) we consider $\rho$ which is not really a density but rather a number of scatterers per unit volume. In the case of a binary mixture, the volume fraction of scatterers is thus simply $\rho v_{i}=\left(\langle N\rangle v_{i}\right) / V=\Phi\left(v_{i}\right.$ is the volume of the scatterer $i$ ). Furthermore, by replacing the isotherm compressibility by the osmotic compressibility (the pressure at work in a binary mixture being the osmotic pressure $\Pi$ ), we obtain finally for the total scattering intensity in turbid media :

$$
\frac{I\left(\boldsymbol{q}, \omega_{f}, R\right)}{I_{0}}=\frac{\pi^{2}}{\lambda_{0}^{4}}\left(\frac{\partial \varepsilon_{r}}{\partial \Phi}\right)^{2} k_{B} T \chi_{T}\left[\frac{1}{1+\left(\frac{q}{q_{0}}\right)^{2}}\right] \frac{V}{R^{2}} \sin ^{2} \varphi
$$




\section{Expression of the scattering force in turbid fluids}

The fraction of scattered intensity in a binary mixture is given by equation (A8) with $q=\left|\boldsymbol{k}_{i}-\boldsymbol{k}_{f}\right|=2 k_{i} \sin (\theta / 2)=$ $\left(4 \pi \sqrt{\varepsilon_{r}} / \lambda_{0}\right) \sin (\theta / 2)$ and $\left(q / q_{0}\right)^{2}=(q \xi)^{2}=\alpha(1-\cos \theta)$, where $\alpha=2\left(2 \pi \sqrt{\varepsilon_{r}} \xi / \lambda_{0}\right)^{2}$ ( $\xi$ being the correlation length of the density fluctuations of the medium). $\theta$ represents the scattering angle whereas $\varphi$ is the angle between the polarization of the incident wave and the direction of the scattered wave vector. One can define as well $\psi$ which reflects the angle between the polarization of the incident wave and the scattering $\operatorname{plane} \operatorname{such} \operatorname{as} \cos \varphi=\cos \psi \sin \theta$. Thus, equation (A8) can be written as:

$$
\frac{I\left(\boldsymbol{q}, \omega_{f}, R\right)}{I_{0}}=A \chi_{T}\left[\frac{1-\cos ^{2} \psi \sin ^{2} \theta}{1+\alpha(1-\cos \theta)}\right] \frac{V}{R^{2}},
$$

with $A=\left(\pi^{2} / \lambda_{0}^{4}\right)\left(\left(\partial \varepsilon_{r} / \partial \Phi\right)\right)^{2} k_{B} T$.

When an incident photon carrying a momentum $\hbar k_{i}$ is elastically scattered by a scatterer of the medium with an angle $\theta$, its momentum in the direction $\widehat{k}_{i}$ decreases by a quantity $\hbar k_{i}(1-\cos \theta)$. This losses of momentum is transferred to the medium giving birth to the scattering force allowing motion of the fluid. Thus, the mean value of momentum lost per photon in the direction $\widehat{k}_{i}$ is given by the integration on the surface of a sphere of radius $R$ of the intensity lost in the direction $\widehat{k}_{i}$ by elastic scattering. This mean value on the surface of radius $R$ with the surface element $R^{2} \mathrm{~d} \psi \sin \theta \mathrm{d} \theta=R^{2} \mathrm{~d} \Omega$ (with $\Omega$ the solid angle) is :

$$
\Delta p_{f}=\hbar k \int_{0}^{2 \pi} \mathrm{d} \psi \int_{-1}^{+1} R^{2} \mathrm{~d}(\cos \theta) \frac{I\left(\boldsymbol{q}, \omega_{f}, R\right)}{I_{0}}(1-\cos \theta) .
$$

After integration and mathematical manipulation, we obtain :

$$
\triangle p_{f}=\hbar k A V \chi_{T} \pi g(\alpha),
$$

with the function :

$$
g(\alpha)=\frac{1}{\alpha^{4}}\left[\frac{8}{3} \alpha^{3}+2 \alpha^{2}+2 \alpha-\left(2 \alpha^{2}+2 \alpha+1\right) \ln (1+2 \alpha)\right] .
$$

If $\mathcal{N}$ represents the number of photons per surface and time units from an incident laser wave, the scattering force per unit volume can be expressed as $f^{s c a t t}=\mathcal{N} \Delta p_{f} / V$. and becomes :

$$
f^{\text {scatt }}=\mathcal{N} h \nu \frac{\sqrt{\varepsilon_{r}}}{c} A \chi_{T} \pi g(\alpha) .
$$

Replacing in equation (A13) $A$ by its expression given above and taking into account that $I_{0}=\mathcal{N} h \nu$, we finally obtain the expression of the scattering force per unit volume applied to a turbid binary liquid mixture as :

$$
f^{\text {scatt }}(r)=\frac{n I_{0}(r)}{c}\left(\frac{\pi^{3}}{\lambda_{0}^{4}}\right)\left(\frac{\partial \varepsilon_{r}}{\partial \Phi}\right)^{2} k_{B} T \chi_{T} g(\alpha),
$$

With $I_{0}(r)$ is the intensity of the incident Gaussian wave with wavelength in vacuum $\lambda_{0}$. Equation (A14) is valid as far as (i) the Ornstein-Zernike approximation is verified and (ii) scattering remains elastic and single. 


\begin{tabular}{ccc}
\hline \hline Setup properties & Temperature dependent variables & Dimensionless numbers \\
\hline & $\xi^{-}=\xi_{0}^{-}\left(\frac{T-T_{c}}{T_{c}}\right)^{-0.63}$ & $\tau=\frac{\omega_{0} \eta_{2}}{\sigma}$ \\
$T c=308 \mathrm{~K}$ & $\chi^{-}=\chi_{0}^{-}\left(\frac{T-T_{c}}{T_{c}}\right)^{-1.24}$ & $U_{\sigma}=\frac{\sigma}{\eta_{2}}$ \\
$\Delta T \in[0.5,1.9] \mathrm{K}$ & $\sigma=\sigma_{0}\left(\frac{T-T_{c}}{T_{c}}\right)^{1.26}$ & $B o=\frac{\omega_{0}{ }^{2}}{l_{c}}$ \\
$\omega_{0} \in[3,9.8] \mu \mathrm{m}$ & $\Delta \rho=\Delta \rho_{0}\left(\frac{T-T_{c}}{T_{c}}\right)^{0.325}$ & $C a_{i}=\frac{2 n_{i} \varpi_{i} P}{\pi c \sigma} \exp ^{-2}$ \\
$\lambda_{0}=532 \mathrm{~nm}$ & $\Delta n=\Delta n_{0}\left(\frac{T-T_{c}}{T_{c}}\right)^{0.325}$ & \\
$P \in[346,1920] \mathrm{mW}$ & $l_{c}=\sqrt{\frac{\sigma}{\left(\rho_{1}-\rho_{2}\right) g}=l_{c 0}\left(\frac{T-T_{c}}{T_{c}}\right)^{0.47}}$ & \\
$\eta_{i}(T)=[1.46-0.014(T-237)]\left(1+2.5 \Phi_{i}\right) .10^{-3}$ & \\
$\Phi_{i=1,2}=\Phi_{0} \pm \Delta \Phi_{0} / 2\left(\left(T-T_{c}\right) / T_{c}\right)^{0.325}$ & $\varpi_{i}=\left(\frac{\pi^{3}}{\lambda_{0}^{4}}\right)\left(\frac{\partial \epsilon_{r i}}{\partial \Phi_{i}}\right)^{2} k_{B} T \chi_{T} f(\alpha)$ & \\
\hline \hline
\end{tabular}

TABLE I. Summarizing table of the physical parameters and dimensionless numbers used in the experiments and simulations. With $\xi_{0}^{-}=2 \mathrm{~nm}, \chi_{0}^{-}=1.34410^{-6} \mathrm{~Pa}^{-1}, \sigma_{0}=510^{-5} \mathrm{~N} \mathrm{~m}^{-1}, \Delta \rho_{0}=53.625 \mathrm{~kg} \mathrm{~m}^{-3}, \Delta n_{0}=-0.0451, l_{c 0}=3.110^{-4} \mathrm{~m}, \Phi_{0}=0.11$ and $\Delta \Phi_{0}=0.275$.

[1] G. Da Costa and J. Calatroni, Transient deformation of liquid surfaces by laser-induced thermocapillarity, Appl. Opt. 18, 233 (1979).

[2] J. P. Longtin, K. H. Jikata, and K. Ogawa, Laser-induced surface-tension-driven flows in liquids, Int. J. Heat Mass Transf. 42, 85 (1999).

[3] S. P. Karlov, D. A. Kazenin, B. I. Myznikova, and I. I. Wertgeim, Experimental and numerical study of the Marangoni convection due to localized laser heating, J. Non-Equilib. Thermodyn. 30, 283 (2005).

[4] H. Chraïbi and J.-P. Delville, Thermocapillary flows and interface deformations produced by localized laser heating in confined environment, Phys. Fluids 24, 032102 (2012).

[5] N. Garnier, R. O. Grigoriev, and M. F. Schatz, Optical Manipulation of Microscale Fluid Flow, Phys. Rev. Lett. 91, 054501 (2003).

[6] M. Robert de Saint Vincent and J.-P. Delville, Thermocapillary migration in small-scale temperature gradients : Application to optofluidic drop dispensing, Phys. Rev. E 85 (2012).

[7] M. Robert de Saint Vincent, H. Chraïbi, and J.-P. Delville, Optical Flow Focusing : Light-Induced Destabilization of Stable Liquid Threads, Phys. Revi. Appl. 4 (2015).

[8] R. T. Mallea, A. Bolopion, J. Beugnot, P. Lambert, and M. Gauthier, Laser-Induced Thermocapillary Convective Flows : A New Approach for Noncontact Actuation at Microscale at the Fluid/Gas Interface, Trans. Mechatronics 22, 693 (2017).

[9] M. A. Kazaryan, N. P. Korotkov, and S. D. Zakharov, Hydrodynamic flows in suspensions caused by powerful pulse-periodic light beams, Phys. Scr. 52, 678 (1995).

[10] A. Savchenko, N. Tabiryan, and B. Zeldovich, Transfer of momentum and torque from a light beam to a liquid, Phys. Rev. E 55, 4773 (1997).

[11] V. G. Shvedov, A. V. Rode, Y. V. Izdebskaya, A. S. Desyatnikov, W. Krolikowski, and Y. S. Kivshar, Giant Optical Manipulation, Phys. Rev. Lett. 105, 118103 (2010).

[12] D. Baigl, Photo-actuation of liquids for light-driven microfluidics: State of the art and perspectives, Lab. Chip. 12, 3637 (2012).

[13] S. Sukhov and A. Dogariu, Non-conservative optical forces, Rep. Prog. Phys. 80, 112001 (2017).

[14] V. Kajorndejnukul, S. Sukhov, and A. Dogariu, Efficient mass transport by optical advection, Scientific Reports 5 (2015).

[15] S. E. Skipetrov, S. S. Chesnokov, S. D. Zakharov, M. A. Kazaryan, and V. A. Shcheglov, Ponderomotive action of light in the problem of multiple scattering of light in a randomly inhomogeneous medium, Jetp Lett. 67, 635 (1998).

[16] S. E. Skipetrov, M. A. Kazaryan, N. P. Korotkov, and S. D. Zakharov, Multiple Light-scattering Probes of Laser-induced Particle Flows in Random Media : Theoretical Consideration, Phys. Scr. 57, 416 (1998).

[17] A. Casner and J.-P. Delville, Laser-Induced hydrodynamic instability of fluid interfaces, Phys. Rev. Lett. 90, 144503 (2003). 
[18] R. D. Schroll, R. Wunenburger, A. Casner, W. W. Zhang, and J.-P. Delville, Liquid Transport due to Light Scattering, Phys. Rev. Lett. 98, 133601 (2007).

[19] R. Wunenburger, B. Issenmann, E. Brasselet, C. Loussert, V. Hourtane, and J.-P. Delville, Fluid flows driven by light scattering, J. Fluid Mech. 666, 273 (2011).

[20] H. Chraïbi, R. Wunenburger, D. Lasseux, J. Petit, and J.-P. Delville, Eddies and interface deformations induced by optical streaming, J. Fluid Mech. 688, 195 (2011).

[21] H. Chraïbi, J. Petit, R. Wunenburger, and J.-P. Delville, Excitation of Fountain and Entrainment Instabilities at the Interface between Two Viscous Fluids Using a Beam of Laser Light, Phys. Rev. Lett. 111, 044502 (2013).

[22] G. Hertz and H. Mende, " der schallstrahlungsdruck in flssigkeiten", Zeitschrift fr Physik A Hadrons and Nuclei 114, 354 (1939)

[23] A. Ashkin and J. M. Dziedzic, "radiation pressure on a free liquid surface", Phys. Rev. Let. 30, 139 (1973).

[24] L. N. Liebermann, The Second Viscosity of Liquids, Phys. Rev. 75, 1415 (1949).

[25] S. Lighthill, Acoustic streaming, J. Sound Vib. 61, 391 (1978).

[26] B. Issenmann, A. Nicolas, R. Wunenburger, S. Manneville, and J.-P. Delville, "deformation of acoustically transparent fluid interfaces by the acoustic radiation pressure", A Letters Journal Exploring the Frotiers of Physics 83, 34002 (2008).

[27] B. T. KhuriYakub, P. A. Reinholdtsen, C. Chou, J. F. Vesecky, and C. C. Teague, Ultrasonic excitation and detection of capillary waves for the measurement of surface film properties, Appl. Phys. Lett. 52, 1571 (1988).

[28] J. B. Lonzaga, D. B. Thiessen, and P. L. Marston, Uniformly valid solution for acoustic propagation in weakly tapered circular waveguides: liquid jet example, J. Acoust. Soc. Am. 124, 151 (2008).

[29] R. Wunenburger, A. Casner, and J.-P. Delville, Light-induced deformation and instability of a liquid interface. I. Statics, Phys. Rev. E 73, 036314 (2006).

[30] . Freysz, Etude des non linarits optiques dans les mlanges liquides binaires critiques, Ph.D. thesis (1990).

[31] A. M. Cazabat, D. Langevin, J. Meunier, and A. Pouchelon, Critical behaviour in microemulsions, J. Phyique Lett. 43, 89 (1982).

[32] B. Jean-Jean, E. Freysz, A. Ducasse, and B. Pouligny, Thermodiffusive and Electrostrictive Optical Nonlinearities in Critical Microemulsions, Europhys. Lett 7, 219 (1988).

[33] N. Bertin, H. Chraibi, R. Wunenburger, J.-P. Delville, and E. Brasselet, Universal Morphologies of Fluid Interfaces Deformed by the Radiation Pressure of Acoustic or Electromagnetic Waves, Phys. Rev. Lett. 109, 244304 (2012).

[34] S. Mitani and K. Sakai, Observation of interfacial tension minima in oil-water-surfactant systems with laser manipulation technique, Faraday Discuss. 129, 141 (2005).

[35] B. J. Berne and R. Pecora, Dynamic light scattering : with applications to chemistry, biology and physics (Dover Publications Inc., 2003). 